\title{
Seiberg-Witten and "Polyakov-like" magnetic bion confinements are continuously connected
}

\author{
Erich Poppitz ${ }^{a}$ and Mithat Ünsal ${ }^{b}$ \\ ${ }^{a}$ Department of Physics, University of Toronto, \\ Toronto, ON M5S 1A\%, Canada \\ ${ }^{b}$ SLAC and Physics Department, Stanford University, \\ Stanford, CA 94025/94305, U.S.A. \\ E-mail: poppitz@physics.utoronto.ca, unsal@slac.stanford.edu
}

ABSTRACT: We study four-dimensional $\mathcal{N}=2$ supersymmetric pure-gauge (Seiberg-Witten) theory and its $\mathcal{N}=1$ mass perturbation by using compactification on $\mathbb{S}^{1} \times \mathbb{R}^{3}$. It is well known that on $\mathbb{R}^{4}$ (or at large $\mathbb{S}^{1}$ size $L$ ) the perturbed theory realizes confinement through monopole or dyon condensation. At small $\mathbb{S}^{1}$, we demonstrate that confinement is induced by a generalization of Polyakov's three-dimensional instanton mechanism to a locally fourdimensional theory - the magnetic bion mechanism - which also applies to a large class of nonsupersymmetric theories. Using a large- vs. small- $L$ Poisson duality, we show that the two mechanisms of confinement, previously thought to be distinct, are in fact continuously connected.

Keywords: Confinement, Supersymmetric gauge theory, Duality in Gauge Field Theories, Solitons Monopoles and Instantons

ARXIV EPRINT: 1105.3969 


\section{Contents}

1 Introduction and results $\quad 2$

1.1 Method 2

1.2 Conclusions 3

1.3 Outline 4

2 Review of the classical $\mathcal{N}=2$ supersymmetric theory 6

2.1 Theory on $\mathbb{R}^{4}$ and global symmetries 6

2.2 Reduction of six dimensional $\mathcal{N}=1$ theory and notation 7

3 Relating 4d monopole particles to 3d monopole-instantons $\quad 8$

3.1 Monopole and dyon particles on $\mathbb{R}^{4} \quad 8$

3.2 Monopole-instantons and dyon-instantons at large $\mathbb{S}^{1} \times \mathbb{R}^{3} \quad 9$

$\begin{array}{lll}3.3 & 3 \mathrm{~d} \text { monopole-instantons at small } \mathbb{S}^{1} \times \mathbb{R}^{3} & 11\end{array}$

3.4 3d-instanton/4d-dyon tower Poisson duality: first pass 15

3.5 General Poisson duality, or 4d dyon spectrum from 3d instanton sums $\quad 17$

$\begin{array}{ll}3.6 & \text { Digression: the meaning of the sum } \\ \end{array}$

4 Mass deformed Seiberg-Witten theory on $\mathbb{S}^{1} \times \mathbb{R}^{3} \quad 21$

4.1 The large- $L$ regime 21

4.2 The small- $L$ regime 24

4.2.1 $\mathcal{N}=1$ perturbation at small $\mathbb{S}^{1} \times \mathbb{R}^{3} \quad 25$

4.2.2 Center-symmetry realization and center-stabilizing bions 28

4.2.3 Chiral symmetry and the topological disorder operator 30

5 Phase diagram and abelian (non-'t Hooftian) large-N limits 31

6 Discussion and open problems 33

A Chirality and fermion zero modes $\quad 35$

A.1 4d-instanton 36

A.2 3d monopole-instanton tower 37

$\begin{array}{lll}\text { A.3 } & \text { Monopole-dyon particle tower } & 38\end{array}$

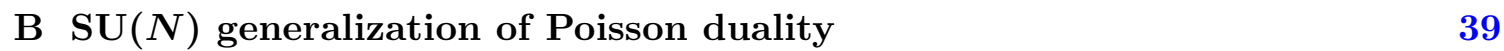




\section{Introduction and results}

In this paper, we study the dynamics of $\mathcal{N}=2$ supersymmetric pure gauge (SeibergWitten [1]) theory and its $\mathcal{N}=1$ mass perturbation compactified on $\mathbb{R}^{3} \times \mathbb{S}^{1}$ through a new method. We mostly work with an $\mathrm{SU}(2)$ gauge group and only mention $\mathrm{SU}(N)$ in connection with non-'t Hooftian (abelian) large- $N$ limits. Ref. [2] already examined this theory on $\mathbb{R}^{3} \times \mathbb{S}^{1}$. A description of the vacuum structure of the theory is given as a function of the circle radius $L$, interpolating between $3 \mathrm{~d}$ and $4 \mathrm{~d}$ results. Supersymmetry, holomorphy, and elliptic curves provide much information about the vacuum of the theory. However, many physical aspects of the mass-perturbed $\mathcal{N}=2$ theory on $\mathbb{R}^{3} \times \mathbb{S}^{1}$ remain open. For example, at small $\mathbb{R}^{3} \times \mathbb{S}^{1}$, one can ask:

i) what generates confinement and the mass gap for gauge fluctuations?

ii) what induces chiral symmetry breaking and generates mass for fermions?

iii) what stabilizes the center symmetry?

These are questions of interests not only in the supersymmetric theory, but also of central importance in non-supersymmetric QCD and QCD-like gauge theories on $\mathbb{R}^{3} \times \mathbb{S}^{1}$. It turns out that adequately answering these questions opens interesting avenues in the study of confinement and topological defects in gauge theories, not exclusively restricted to supersymmetric theories.

\subsection{Method}

In this work, we use a different methodology relative to ref. [2] to study the theory on $\mathbb{R}^{3} \times \mathbb{S}^{1}$. Our approach, shown in the commutative diagram in figure 1 , permits us to study this theory by using simple field theory techniques. Some of our techniques also apply to non-supersymmetric theories.

Let us now briefly describe the physics of Seiberg-Witten solution at $\mathbb{R}^{4}$ and the reasoning behind figure 1 . The $\mathcal{N}=2$ theory on $\mathbb{R}^{4}$ possesses a quantum moduli space parameterized by $u=\left\langle\operatorname{tr} \Phi^{2}\right\rangle$, where $\Phi$ is an adjoint chiral multiplet. The $u$-modulus also provides a control parameter. On the moduli space, the $\mathrm{SU}(2)$ gauge symmetry is Higgsed

down to $\mathrm{U}(1)$ at a scale $|u|^{\frac{1}{2}}$. Since the theory is asymptotically free, for $|u| \gg \Lambda_{\mathcal{N}=2}^{2}$, where $\Lambda_{\mathcal{N}=2}$ is the strong scale, the theory is (electrically) weakly coupled, $g_{4}^{2}(|u|) \ll 1$. (From now on, we set $\Lambda_{\mathcal{N}=2}=1$ ). The $|u| \lesssim 1$ domain (shaded region in figure 1) is electrically strongly coupled.

The $\mathrm{SU}(2) \rightarrow \mathrm{U}(1)$ theory possesses the 't Hooft-Polyakov monopole and dyon particles, which are heavy at $|u| \gg 1$. There are two points on the moduli space in the shaded region in figure 1 , where a monopole $(u=+1)$ or a dyon $(u=-1)$ become massless. The low-energy limit of the theory near the monopole (or dyon) points is described by the $\mathcal{N}=2$ supersymmetric electrodynamics (SQED) of massless monopoles (or dyons). The gauge field and the coupling in SQED are dual to the ones in the microscopic theory. In particular, whenever the electric coupling is large, the dual magnetic coupling is small and vice versa. The effective field theory descriptions near the $u=+1$ and $u=-1$ points are 


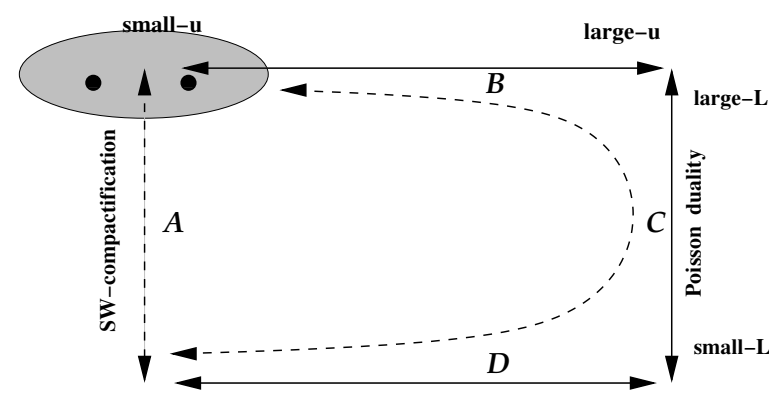

Figure 1. Taking different paths in the $u-L$ plane. The horizontal direction, $u$, is the modulus of Seiberg-Witten theory and the vertical, $L$, is the size of $\mathbb{S}^{1}$. Ref. [2] studied the softly broken $\mathcal{N}=2$ theory on $\mathbb{R}^{3} \times \mathbb{S}^{1}$ by using elliptic curves through path $A$. In this work, we reexamine the same theory along the path $B C D$ in moduli space. The $C D$ branch always remains semi-classical and allows us to understand the relation between the topological defects responsible for confinement at small- $L$ and large- $L$ in detail.

mutually non-local and there is no global macroscopic theory which describes both $u=+1$ and $u=-1$. Physically, one of the most interesting outcomes of the Seiberg-Witten solution is that when the $\mathcal{N}=2$ theory is perturbed by an $\mathcal{N}=1$ preserving mass term for $\Phi$, it exhibits confinement of electric charges due to magnetic monopole or dyon condensation.

Ref. [2] studied the $\mathcal{N}=2 \mathrm{SYM}$ and its softly broken $\mathcal{N}=1$ version on $\mathbb{R}^{3} \times \mathbb{S}^{1}$ by using elliptic curves through path- $A$ on figure 1 . However, if we would like to understand the relation between the topological defects (and field theories) at large and small $\mathbb{S}^{1}$, there are some intrinsic difficulties associated with path- $A$. In particular, the large- $\mathbb{S}^{1}$ theory is magnetically weakly and electrically strongly coupled, and the small- $\mathbb{S}^{1}$ one is electrically weakly (by asymptotic freedom) and magnetically strongly coupled. Thus, when $L \sim 1$ and $|u| \lesssim 1$, both electric and magnetic couplings are order one, and we do not know how to address this domain in field theory. To avoid this difficulty, we propose a compactification (path- $C$ ) at large- $u$ where the theory is always electrically weakly coupled, regardless of the $\mathbb{S}^{1}$-size $L$. Path- $D$ is also always weakly coupled, either because the $u$-modulus is large or because an additional modulus, the Wilson line along $\mathbb{S}^{1}$, is turned on (also note that in the small- $L$ domain the $\mathcal{N}=2$ theory always abelianizes, and the long-distance dynamics is described by a three dimensional hyper-Kähler nonlinear sigma model [2]).

\subsection{Conclusions}

We find, by using the techniques of ref. [2] and of our current work, that a locally four dimensional generalization [3, 4] of Polyakov's 3d instanton mechanism of confinement [5] takes over in the small- $L$ mass-perturbed $\mathcal{N}=2$ theory. To elucidate, note that the theory possesses 3d instanton (and anti-instanton) solutions, which, when embedded in $\mathbb{R}^{3} \times \mathbb{S}^{1}$, have magnetic, $Q_{m}=\int_{\mathbb{S}_{\infty}^{2}} F$, and topological, $Q_{T}=\int_{\mathbb{R}^{3} \times \mathbb{S}^{1}} F \widetilde{F}$ charges, normalized to $\left(Q_{m}, Q_{T}\right)= \pm\left(1, \frac{1}{2}\right)$. There are also twisted instantons (and anti-instantons), which carry charges $\left(Q_{m}, Q_{T}\right)= \pm\left(-1, \frac{1}{2}\right)$. The mass gap for gauge fluctuations and confinement in the mass-perturbed $\mathcal{N}=2$ theory arise due to Debye screening by topological defects with charges $\left(Q_{m}, Q_{T}\right)=( \pm 2,0)$. This mechanism of confinement was called the "magnetic 
bion" mechanism in $[3,4]$ and we show here that it also takes place in the $\mathcal{N}=1$ mass deformation of Seiberg-Witten theory at small $L$. The fact that the leading instanton amplitude in the semi-classical expansion cannot generate mass gap for gauge fluctuation which distinguishes the magnetic bion mechanism from Polyakov's $3 \mathrm{~d}$ instanton mechanism - is due to the presence of fermion zero modes, dictated by the Nye-Singer index theorem for the Dirac operator on $\mathbb{S}^{1} \times \mathbb{R}^{3}[6,7]$.

Our main conclusions are:

1. There are two types of confinement mechanisms in mass-perturbed $\mathcal{N}=2$ theory. At $L$ large compared to the inverse strong scale of the theory, confinement is due to magnetic monopole or dyon condensation. At small $L$, it is the "Polyakov-like" magnetic bion mechanism briefly described above.

2. Under the reasonable assumption that supersymmetric theories with supersymmetrypreserving boundary conditions on $\mathbb{S}^{1} \times \mathbb{R}^{3}$ do not have any phase transition as a function of radius, these two mechanisms ought to be continuously connected. The physical questions we address in this work are:

(a) What is the relation between the monopole and dyon particles on $\mathbb{R}^{4}$ (or large $\mathbb{S}^{1} \times \mathbb{R}^{3}$ ) and the monopole-instantons and magnetic bion-instantons of the small $\mathbb{S}^{1} \times \mathbb{R}^{3}$ regime? How do we relate the two confinement mechanisms?

(b) What is the region of validity of the various small- and large- $L$ descriptions?

Our results show that the relation between the topological defects responsible for confinement at small $L$ and large $L$ is intricate - even in the case where confinement remains manifestly abelian at any $L$, as in the mass-perturbed Seiberg-Witten theory. However, along the path- $C$ in figure 1 of undeformed theory, we find a precise duality relation between the semi-classical topological defects pertinent to confinement at large- and small- $L$. More precisely, the $3 \mathrm{~d}$ monopole-instantons and twisted monopole-instantons, which make up the magnetic bion "molecules" that generate mass gap and confinement at small $L$, have a Kaluza-Klein tower. The nonperturbative contribution of this tower is dual, through a Poisson resummation, to that of the tower of $4 \mathrm{~d}$ monopole/dyon particles whose Euclidean worldlines wrap around the compact direction. We refer to the duality along the path- $C$ as Poisson duality. This duality presents an explicit relation between the topological defects responsible for confinement at small $\mathbb{R}^{3} \times \mathbb{S}^{1}$ and on $\mathbb{R}^{4}$.

3. The magnetic bion mechanism also holds in a large-class of non-supersymmetric theories at small $L$. Thus, our construction gives a map between theoretically controllable confinement mechanisms in non-supersymmetric and supersymmetric gauge theories.

\subsection{Outline}

We begin in section 2 by reviewing the classical pure $\mathcal{N}=2$ supersymmetric Yang-Mills theory, both using $4 \mathrm{~d}$ notation (section 2.1) and dimensional reduction from $6 \mathrm{~d}$ (section 2.2). 
The latter is useful when studying the supersymmetries preserved by various classical solutions in appendix A. In section 2.2, we also introduce come useful notation.

We begin the discussion of the classical solutions in section 3 by recalling, in section 3.1, the properties of monopole and dyon particles on $\mathbb{R}^{4}$. The corresponding tower of monopoleand dyon-instantons on $\mathbb{R}^{3} \times \mathbb{S}^{1}$, pertinent to the large- $L$ nonperturbative dynamics, is constructed in section 3.2. In section 3.3, we describe the tower of winding monopoleinstanton solutions at a generic point in moduli space, relevant to the small- $L$ dynamics. The Poisson duality between the $3 \mathrm{~d}$ tower of winding solutions and $4 \mathrm{~d}$ tower of dyoninstantons is discussed in the following three sections. In section 3.4, the duality is discussed and qualitatively explained in a simplifying limit. In section 3.5, a more general duality relation is derived and then discussed in section 3.6.

In section 4, we study the role the winding monopole-instantons and dyon-instantons, discussed above, play in the nonperturbative dynamics of confinement and chiral symmetry breaking at large or small $L$. In section 4.1, we recall the SW description of monopole/dyon condensation in the mass-perturbed theory and give the large- $L$ expressions for the mass gap and string tension. Then, we explain the difficulties a compactification along path A of figure 1 would face. We also give an effective $3 \mathrm{~d}$ description of the physics at scales larger than $L$, valid for $L \Lambda_{\mathcal{N}=2} \gg 1$, using a chain of known 3 d dualities.

The small- $L$ dynamics is studied in section 4.2 , beginning with a discussion of the 't Hooft vertices induced by the winding monopole-instantons of lowest action. Then, in section 4.2.1, we explain the effect of the $\mathcal{N}=1$ preserving mass perturbation, the generation of a superpotential, the resulting vacua of the theory, and give expressions for the mass gap and string tension (all results well-known in the literature). Then, we concentrate on the physical mechanisms responsible for the center-symmetry stabilization and confinement. We note that they are due to different kinds of instanton-anti-instanton "molecules". Most notably, we explain that the mass of the dual photon is generated by magnetic bions, bound states of monopoles and twisted anti-monopoles of magnetic charge two, and an example of a topological "molecule" whose stability is semiclassically calculable. The realization of the unbroken center-symmetry is discussed in section 4.2.2. The physics of chiral symmetry breaking is discussed in section 4.2.3, along with an elaboration on some imprecise statements in the literature.

In section 5 , we discuss the phase diagram in the $m-L$ plane, indicating the regimes where the different topological excitations discussed above play a role in the confinement mechanism. We consider both the $\mathrm{SU}(2)$ ( $\operatorname{small}-N$ ) and large- $N$ cases. In the latter case, we note that the abelian description of the dynamics persists as $N \rightarrow \infty$ only in non 't Hooftian large- $N$ limits, both at small $L$ (where $L$ must scale as $1 / N$ ) and large $L$ (where the soft breaking mass $m \sim 1 / N^{4}$ ).

We summarize our findings and discuss some open problems in section 6 . We give various technical details in the appendices. In appendix A, we study the supersymmetries preserved by the various solutions discussed in the paper, in order to identify the nature of the unlifted supersymmetric fermion zero modes. In appendix B, we generalize to $\mathrm{SU}(N)$ the Poisson duality relation of section 3.5. 


\section{Review of the classical $\mathcal{N}=2$ supersymmetric theory}

\subsection{Theory on $\mathbb{R}^{4}$ and global symmetries}

The matter content and bosonic symmetries of the pure $\mathcal{N}=2$ supersymmetric Yang-Mills theory on $\mathbb{R}^{4}$ fill a representation of the $\mathrm{SO}(4) \sim\left[\mathrm{SU}(2)_{\mathcal{L}} \times \mathrm{SU}(2)_{\mathcal{R}}\right]_{E}$ Euclidean Lorentz symmetry and the $\mathrm{SU}(2)_{R} \times \mathrm{U}(1)_{R}$ chiral R-symmetry. The transformation properties under $\left[\mathrm{SU}(2)_{\mathcal{L}} \times \mathrm{SU}(2)_{\mathcal{R}} \times \mathrm{SU}(2)_{R}\right]_{\mathrm{U}(1)_{R}}$ are as follows: the gauge field $A_{\mu} \sim\left(\frac{1}{2}, \frac{1}{2}, 0\right)_{0}$, the scalar $\phi \sim(0,0,0)_{+2}$, fermions $\lambda^{i} \sim\left(\frac{1}{2}, 0, \frac{1}{2}\right)_{+1}$. All fields are valued in the adjoint representation of the gauge group $G$ and fill a gauge multiplet of $\mathcal{N}=2$ supersymmetry. In this paper, we study mostly $G=\mathrm{SU}(2)$ and give a generalization to $\mathrm{SU}(N)$ for some of the results.

The $\mathcal{N}=2$ supersymmetric gauge multiplet diamond can be decomposed in terms of $\mathcal{N}=1$ multiplets, vector $V=\left(A_{\mu}, \lambda\right)$ and chiral $\Phi=(\phi, \psi)$ multiplets, as well as $\mathcal{N}=1^{\prime}$ $V^{\prime}=\left(A_{\mu}, \psi\right), \Phi^{\prime}=(\phi, \lambda)$ multiplets as shown below:

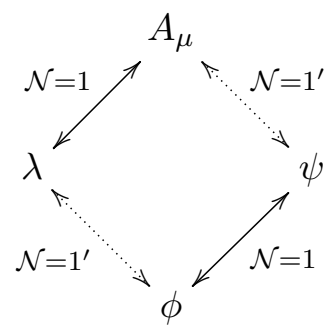

We will eventually be interested in the theory with only $\mathcal{N}=1$ supersymmetry, where $\mathcal{N}=1^{\prime}$ part of the supersymmetry is broken by a soft mass term for the $\Phi$-multiplet. We parameterize the $\mathrm{SU}(2)_{R}$ doublet as $\left(\begin{array}{c}\lambda^{1} \\ \lambda^{2}\end{array}\right)=\left(\begin{array}{l}\lambda \\ \psi\end{array}\right)$, where the first form is used whenever we want to make $\mathrm{SU}(2)_{R}$ invariance manifest.

The Lagrangian of $\mathcal{N}=2$ Yang-Mills theory may be written in component fields as:

$\mathcal{L}=\frac{2}{g_{4}^{2}} \operatorname{tr}\left[\frac{1}{4} F_{\mu \nu}^{2}+D_{\mu} \phi^{\dagger} D_{\mu} \phi+\frac{1}{2}\left[\phi^{\dagger}, \phi\right]^{2}+i \bar{\lambda}_{i} \bar{\sigma}_{\mu} D_{\mu} \lambda^{i}-\frac{i}{\sqrt{2}} \epsilon_{i j} \lambda^{i}\left[\lambda^{j}, \phi^{\dagger}\right]-\frac{i}{\sqrt{2}} \epsilon^{i j} \bar{\lambda}_{i}\left[\bar{\lambda}_{j}, \phi\right]\right]$,

where $D_{\mu}=\partial_{\mu}+i\left[A_{\mu},\right]$ and the field strength is $F_{\mu \nu}=\partial_{\mu} A_{\nu}-\partial_{\nu} A_{\mu}+i\left[A_{\mu}, A_{\nu}\right]$. We normalize the Lie algebra generators as $\operatorname{tr} t^{a} t^{b}=\frac{1}{2} \delta^{a b}$. The component formalism makes the chiral symmetries manifest, but it hides the exact $\mathcal{N}=2$ supersymmetry.

The classical $\mathrm{U}(1)_{R}$ symmetry is anomalous quantum mechanically. For an $\mathrm{SU}(N)$ gauge group, $4 \mathrm{~d}$ instantons generate an amplitude:

$$
e^{-S_{I}}\left[\frac{1}{2} \epsilon_{i_{1} i_{2}} \epsilon_{j_{1} j_{2}}\left(\lambda^{i_{1}} \lambda^{j_{1}}\right)\left(\lambda^{i_{2}} \lambda^{j_{2}}\right)\right]^{N} \equiv e^{-S_{I}}\left[\operatorname{det}_{i, j}\left(\lambda^{i} \lambda^{j}\right)\right]^{N},
$$

which is manifestly invariant under $\mathrm{SU}(2)_{\mathcal{R}}$, but rotates by a phase $e^{i 4 N \alpha}$ under $\mathrm{U}(1)_{R}$, $\lambda^{i} \rightarrow e^{i \alpha} \lambda^{i}$. Thus, the quantum theory respects only a $\mathbb{Z}_{4 N}$ subgroup of $\mathrm{U}(1)_{R}$. For $\mathrm{SU}(2)$ gauge group, the exact chiral symmetry of the quantum theory is:

$$
\left(\mathrm{SU}(2)_{R} \times \mathbb{Z}_{8}\right) / \mathbb{Z}_{2}
$$


where $\mathbb{Z}_{2}$ is factored out to prevent double counting of the factor $(-1)^{F}$ (where $F$ is fermion number) common to the center of SU(2) $R$ and $\mathbb{Z}_{8}$.

Since the $\mathcal{N}=2$ Lagrangian (2.2) includes terms of the form $\phi^{\dagger}\left[\lambda_{1}, \lambda_{2}\right]$, the scalar $\phi$ is also charged under $\mathbb{Z}_{8}$. It transforms as $\phi \rightarrow e^{i \frac{\pi}{2}} \phi$. The simplest gauge invariant that we build out of $\phi$ is $u \equiv \operatorname{tr} \phi^{2}$. The $u$ field parametrizes the classical moduli space of gauge theory, and it changes sign under the $\mathbb{Z}_{8}$ action. This discrete symmetry will be crucial once we consider the theory on $\mathbb{R}^{3} \times \mathbb{S}^{1}$. To summarize, the action of the anomaly-free chiral $\mathbb{Z}_{8}$ symmetry is:

$$
\mathbb{Z}_{8}: \lambda^{i} \rightarrow e^{i \frac{2 \pi}{8}} \lambda^{i}, \quad \phi \rightarrow e^{i \frac{4 \pi}{8}} \phi, \quad u \rightarrow-u .
$$

Note that the $\mathbb{Z}_{8}$ symmetry is unbroken only at the $u=0$ point in the classical moduli space. This will also have interesting consequences for the theory on $\mathbb{R}^{3} \times \mathbb{S}^{1}$.

\subsection{Reduction of six dimensional $\mathcal{N}=1$ theory and notation}

It will be also useful to describe the $\mathcal{N}=2$ theory in $4 \mathrm{~d}$ by starting with the minimal supersymmetric Yang-Mills theory in 6d with the Lagrangian:

$$
\mathcal{L}=\frac{2}{g_{4}^{2}} \operatorname{tr}\left[\frac{1}{4} F_{M N}^{2}+i \bar{\Psi} \Gamma_{M} D_{M} \Psi\right]
$$

where $M, N=1, \ldots, 6 . \Gamma^{M}, M=1, \ldots 6$ denote the six gamma-matrices satisfying the Clifford algebra $\left\{\Gamma^{M}, \Gamma^{N}\right\}=2 \delta_{M N}$, and $\Gamma^{7}$ is the chirality matrix in six dimensions. ${ }^{1}$ The complex spinor $\Psi$ satisfies the chirality condition, $\left(\Gamma_{7}+1\right) \Psi=0$.

The $\mathcal{N}=1$ theory in $6 \mathrm{~d}$ has an $\mathrm{SU}(2)_{R}$ chiral symmetry which acts on fermions (it is not manifest in the way we have written it). The fermions as well as the supercharges transform as doublets under this symmetry, whereas the gauge field is a singlet. Dimensional reduction turns the Lorentz symmetry in the reduced directions into global $R$-symmetries of the lower dimensional theory. Let us denote the Euclidean spacetime directions as $x^{1,2,3,4,5,6}$. The $\mathcal{N}=2$ theory on $\mathbb{R}^{4}$ may be obtained by erasing the $x^{5,6}$ dependence from all fields. This means that the $\mathrm{SO}(6)_{E}$ Euclidean Lorentz symmetry transmutes to $\mathrm{SO}(4)_{E} \times \mathrm{SO}(2)_{R}$ symmetry, whose covering group is $[\mathrm{SU}(2) \times \mathrm{SU}(2)]_{E} \times \mathrm{U}(1)_{R}$. Together with the $\mathrm{SU}(2)_{R}$ mentioned above, this is the symmetry group of the $4 \mathrm{~d} \mathcal{N}=2$ theory described in section 2.1 .

Let us denote the gauge field of the six dimensional theory as $A^{M}$. Consider both dimensional reduction and compactification ${ }^{2}$ down to to $\mathbb{R}^{4}, \mathbb{R}^{3} \times \mathbb{S}^{1}$, and $\mathbb{R}^{3}$. The $6 \mathrm{~d}$

\footnotetext{
${ }^{1}$ We may use the following basis for computations:

$$
\Gamma^{\mu}=\sigma_{2} \otimes \gamma_{\mu}, \quad \Gamma^{5}=\sigma_{2} \otimes \gamma_{5}, \quad \Gamma^{6}=\sigma_{1} \otimes 1_{4}, \quad \Gamma^{7}=\sigma_{3} \otimes 1_{4},
$$

where $\gamma_{1, \ldots, 4}$ are four-dimensional gamma matrices and $\gamma_{5}=\gamma_{1} \ldots \gamma_{4}$. However, using an explicit basis is not necessary for our purposes, see appendix A.

${ }^{2}$ We distinguish dimensional reduction and compactification. Compactification, unlike dimensional reduction, does not alter the microscopic chiral symmetries of the theory, which has important consequences.
} 
gauge field decomposes as follows:

$$
\begin{array}{lll}
A_{M} \rightarrow A_{\mu} \oplus \underbrace{A_{5}, A_{6}}_{\phi, \phi^{\dagger}}, & \mu=1, \ldots, 4, & \mathbb{R}^{4} \\
A_{M} \rightarrow A_{i} \oplus \underbrace{A_{4}}_{b} \oplus \underbrace{A_{5}, A_{6}}_{\phi, \phi^{\dagger}}, & i=1,2,3, & \mathbb{R}^{3} \times \mathbb{S}^{1}
\end{array}
$$

where $\phi=\left(A_{5}+i A_{6}\right) / \sqrt{2}$. If the gauge theory abelianizes, at scales larger than the $\mathbb{S}^{1}$ size $L$ we can dualize the three dimensional field strength to a compact scalar $\sigma$, via:

$$
F_{i j}=\frac{g_{4}^{2}}{4 \pi L} \epsilon_{i j k} \partial_{k} \sigma
$$

and use the complex fields $B=(b+i \sigma) / \sqrt{2}$ to obtain the decomposition:

$$
A_{M} \rightarrow \underbrace{\sigma, b}_{B, B^{\dagger}} \oplus \underbrace{A_{5}, A_{6}}_{\phi, \phi^{\dagger}}, \quad b \equiv \frac{4 \pi}{g_{4}^{2}} \omega, \quad \omega \equiv L A_{4} \quad \mathbb{R}^{3} .
$$

In (2.11) $A_{4}$ refers to the value of $A_{4}$ in the Cartan subalgebra (the unbroken abelian gauge group) and $\omega$ denotes the corresponding Wilson line around the compact direction.

\section{Relating 4d monopole particles to $3 \mathrm{~d}$ monopole-instantons}

\subsection{Monopole and dyon particles on $\mathbb{R}^{4}$}

Consider the pure $\mathcal{N}=2$ theory in the semi-classical domain of its moduli space. Denoting $v=|u|^{\frac{1}{2}}$, this is the regime where $v \gg 1$; recall that we set $\Lambda_{\mathcal{N}=2}=1$. BPS particles with electric and magnetic charges $\left(n_{m}, n_{e}\right)$ have masses determined by the central charge $Z_{\left(n_{m}, n_{e}\right)}=v\left(n_{e}+n_{m} \tau\right)$, where $\tau=\frac{4 \pi i}{g_{4}^{2}}+\frac{\theta}{2 \pi}$ is the holomorphic gauge coupling:

$$
M\left(n_{m}, n_{e}\right)=\left|Z_{\left(n_{m}, n_{e}\right)}\right|=v \sqrt{n_{m}^{2}\left(\frac{4 \pi}{g_{4}^{2}}\right)^{2}+\left(n_{e}+n_{m} \frac{\theta}{2 \pi}\right)^{2}}=v \sqrt{n_{m}^{2}\left(\frac{4 \pi}{g_{4}^{2}}\right)^{2}+n_{e}^{2}} .
$$

From now on, we set $\theta=0$.

In the limit $v \gg 1$, the coupling is small $g_{4}^{2}(v) \ll 1$. The monopole with $n_{m}=1$ and arbitrary $n_{e}$ will be relevant below. Its mass is given by expanding (3.1):

$$
M\left(1, n_{e}\right) \approx \frac{4 \pi v}{g_{4}^{2}}+\frac{1}{2} \frac{g_{4}^{2} v}{4 \pi} n_{e}^{2}=M_{(1,0)}+\left(M_{\left(1, n_{e}\right)}-M_{(1,0)}\right) \equiv M_{(1,0)}+\Delta M_{\left(1, n_{e}\right)} .
$$

This formula has a well-known physical interpretation. A monopole in the four-dimensional theory has four collective coordinates. The classical solution is not invariant under three spatial translations and under the unbroken subgroup $\mathrm{U}(1)_{e} \subset \mathrm{SU}(2)$. The corresponding collective coordinate space is $(\vec{a}, \varphi) \in \mathbb{R}^{3} \times \mathbb{S}_{\varphi}^{1}$. The angular zero mode is generated by $\mathrm{U}(1)_{e}$ rotations and the eigenvalue of rotation on the unit circle $\mathbb{S}_{\varphi}^{1}$ is the electric charge. The wave function associated with the collective coordinates is $\Psi(\vec{a}, \varphi)=e^{i \vec{p} \cdot \vec{a}} e^{i n_{e} \varphi}$. When we quantize the BPS monopole with $n_{m}=1$, we observe that it can carry arbitrary electric 

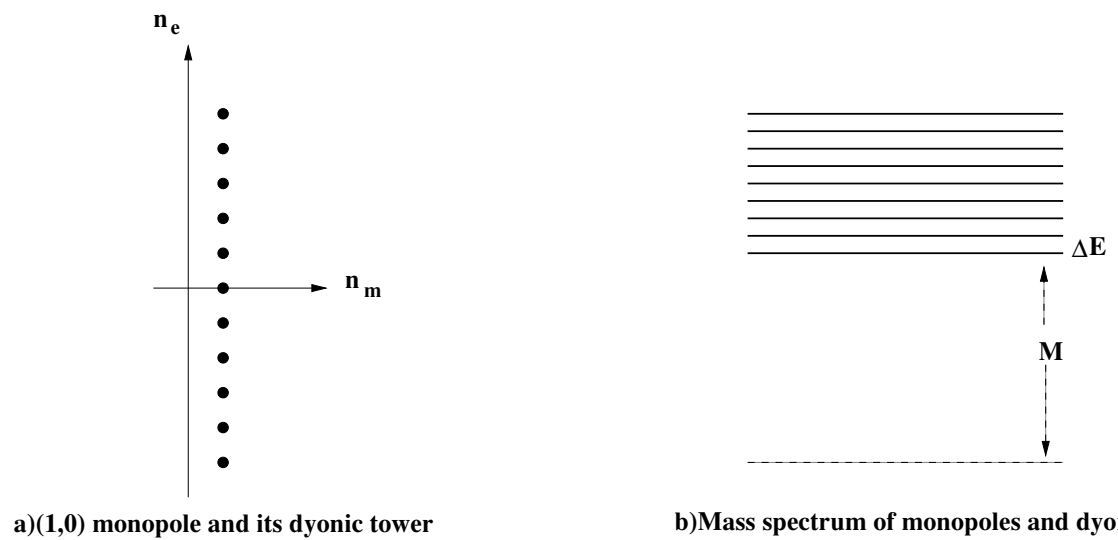

b)Mass spectrum of monopoles and dyons

Figure 2. a) The spectrum of charges of a monopole and its dyonic tower obtained by quantizing the $\mathrm{U}(1)_{e}$ zero mode. b) The mass spectrum. In the semi-classical regime, $\Delta E \equiv E_{(1, \pm 1)}-E_{(1,0)} \ll M$. This tower of states, labelled by electric charge, is pertinent to large- $L$ and is Poisson-dual to the 3d BPS monopole-instanton and its tower, characterized by the winding number, and pertinent to small- $L$.

charge, an integer multiple of the fundamental charge, as shown in figure 2a (such towers exist for any magnetic charge monopole $\left(n_{m}, 0\right)$, albeit they may be unstable). The dyonic tower of the anti-monopole is $\left(-1, n_{e}\right), n_{e} \in \mathbb{Z}$.

In the semi-classical regime, the mass of a $(1,1)$ dyon (3.2) differs negligibly from that of a monopole. In fact, a large number of states occupying the dense band shown in figure 2 (clearly, the spectrum is not equidistant as may appear from the figure) has $\Delta M_{\left(1, n_{e}\right)} \ll$ $M_{(1,0)}$. The states almost degenerate with the monopole have $\left|n_{e}\right| \ll n_{e}^{\max } \ll 4 \pi / g_{4}^{2}$. When $\left|n_{e}\right|<n_{e}^{\max }$, the fermionic zero modes of the states in the tower will also be identical, at leading order; this will be important for our future considerations.

Also for future reference we note that the Bogomolnyi's bound applied to $\left(1, n_{e}\right)$ BPS monopole/dyon particles yield the first order differential equations (see ref. [8] for a review):

$$
\begin{aligned}
\vec{B}-\cos \delta_{n_{e}} \vec{D} A_{5} & =0, \\
\vec{E}-\sin \delta_{n_{e}} \vec{D} A_{5} & =0, \\
D_{4} A_{5} & =0,
\end{aligned}
$$

where:

$$
e^{i \delta_{n_{e}}}=\frac{\frac{4 \pi}{g_{4}^{2}}+i n_{e}}{\sqrt{\left(\frac{4 \pi}{g_{4}^{2}}\right)^{2}+n_{e}^{2}}} .
$$

We have rotated the scalar vev, see $(2.8)$, in the $\phi=\left(A_{5}, A_{6}\right)$ plane to purely $A_{5}$, with no loss of generality.

\subsection{Monopole-instantons and dyon-instantons at large $\mathbb{S}^{1} \times \mathbb{R}^{3}$}

To study the theory on $\mathbb{S}^{1} \times \mathbb{R}^{3}$, we compactify the $x_{4}$ direction on a circle with circumference $L$. When $L \Lambda_{\mathcal{N}=2} \gg 1$, the spectrum of the theory is clearly that of Seiberg-Witten theory on $\mathbb{R}^{4}$ with trivial restrictions due to the boundary condition in $x_{4}$. The perturbative 
spectrum consists of photons, electrically charged $W$-bosons, and their superpartners, while the non-perturbative spectrum is comprised of monopoles, dyons, and their superpartners.

In the $v \gg 1$ regime, the nonperturbative magnetically charged states are semiclassically accessible and are thus significantly heavier then the perturbative states. They do, however, contribute to the dynamics of the quantum theory on $\mathbb{S}^{1} \times \mathbb{R}^{3}$. If one lets the Euclidean worldline of a monopole/dyon particle wrap around the $\mathbb{S}^{1}$, this "pseudo-particle" acquires a finite Euclidean action, $S\left(n_{m}, n_{e}\right)=L M\left(n_{m}, n_{e}\right)$. This means that it has to be interpreted not as a (BPS) state in the compactified theory, but rather as an instanton of action, which, for $n_{m}=1$, is given by: ${ }^{3}$

$$
S\left(1, n_{e}\right)=L M\left(1, n_{e}\right)=v L \sqrt{\left(\frac{4 \pi}{g_{4}^{2}}\right)^{2}+n_{e}^{2}} .
$$

These instantons represent saddle points of the Euclidean path integral and their contributions must be summed over.

When we consider the theory on $\mathbb{R}^{4}$, we can gauge away the gauge field in any one chosen direction, in particular its $x_{4}$-component $A_{4}$. However, once the theory is compactified, $x_{4} \equiv x_{4}+L$, the zero mode of $A_{4}$ - equivalently, the Wilson line around $\mathbb{S}^{1}$ - can no longer be gauged away. In the supersymmetric theory on $\mathbb{S}^{1} \times \mathbb{R}^{3}$, we are free to turn on an arbitrary constant and homogeneous $A_{4}$ background gauge field commuting with the vev of $\phi, A_{4}=a_{4} T^{3}$. This background gauge field naturally couples to the electric charge of the dyonic tower, modifying the action:

$$
S\left(1, n_{e}\right) \rightarrow S\left(1, n_{e}\right)+i n_{e} \int_{0}^{L} d x^{4} a_{4}=S\left(1, n_{e}\right)+i n_{e} a_{4} L=S\left(1, n_{e}\right)+i n_{e} \omega,
$$

where in the last line we recalled the definition (2.11) of $\omega$, an angular variable (see the following section 3.3).

The "dyon-instantons" with charges $\left(1, n_{e}\right)$ induce amplitudes $\sim e^{-S\left(1, n_{e}\right)} e^{i \sigma+i n_{e} \omega} \times$ (fermion zero modes) in the long distance effective Lagrangian, where $\sigma$ is the dual photon defined in (2.10). (The fermionic zero modes are discussed in sections 3.4, 3.5, and appendix A.) The $e^{i \sigma}$ factor, within the dilute gas approximation, takes into account the long-distance Coulomb interactions between dyon-instantons [5]. Thus, in the large- $L$ limit, the sum of the leading semiclassical contributions with magnetic charge $n_{m}=1$ comes from the infinite dyonic tower $\left(1, n_{e}\right), n_{e} \in \mathbb{Z}$. This gives, schematically:

$$
\text { (Dyon sum at large- } L) \sim e^{i \sigma} \sum_{n_{e} \in \mathbb{Z}} e^{-v L \sqrt{\left(\frac{4 \pi}{g_{4}^{2}}\right)^{2}+n_{e}^{2}}+i n_{e} \omega} \text {. }
$$

In the semiclassical domain $g_{4}^{2}(v) \ll 1$, we use $(3.2)$ to obtain:

$$
\text { (Dyon sum at large- } L \text { ) } \sim e^{i \sigma} e^{-\frac{4 \pi v L}{g_{4}^{2}}} \sum_{n_{e} \in \mathbb{Z}} e^{-\frac{1}{2} \frac{v L g_{4}^{2}}{4 \pi} n_{e}^{2}+i n_{e} \omega}
$$

\footnotetext{
${ }^{3}$ The theory also has $4 \mathrm{~d}$ instantons, obeying the self-duality condition $F_{\mu \nu}=\frac{1}{2} \epsilon_{\mu \nu \rho \sigma} F^{\rho \sigma}$ of action $\frac{8 \pi^{2}}{g_{4}^{2}}$. As these carry no magnetic charge, they are not relevant for confinement at small $L$.
} 
The sum over electric charges in (3.8) converges rather fast for $\frac{v L g_{4}^{2}}{4 \pi} \gg 1$, i.e., at large$L$, and the first few terms in the sum are sufficient to produce accurate semi-classical results. Conversely, this sum converges very slowly if $\frac{v L g_{4}^{2}}{4 \pi} \ll 1$, where a more convergent description, as will be described in the next subsection, emerges - this time in terms of $3 \mathrm{~d}$ monopole-instantons and twisted monopole-instantons.

\subsection{3d monopole-instantons at small $\mathbb{S}^{1} \times \mathbb{R}^{3}$}

Consider again the Euclidean action (2.2) of the $\mathcal{N}=2$ gauge theory on $\mathbb{R}^{3} \times \mathbb{S}^{1}$ and use $A_{5}, A_{6}$ to denote the scalar $\phi$, see (2.11). $A_{5}$ and $A_{6}$ can be rotated to each other, by using the symmetries (in what follows we will use this to set $\left\langle A_{6}\right\rangle=0$ ), but not to $A_{4}$ due to the lack of any symmetry relating them. Thus, we take only $A_{4}$ and $A_{5}$ to have nonzero vevs:

$$
\left\langle A_{5}\right\rangle=a_{5} T_{3} \equiv v T_{3}=\left[\begin{array}{cc}
\frac{v}{2} & 0 \\
0 & -\frac{v}{2}
\end{array}\right], \quad\left\langle A_{4}\right\rangle=a_{4} T_{3} \equiv \frac{\omega}{L} T_{3}=\left[\begin{array}{cc}
\frac{\omega}{2 L} & 0 \\
0 & -\frac{\omega}{2 L}
\end{array}\right] .
$$

Further, we note that in the compactified theory the vev $a_{4}$ is actually an angular variable, ${ }^{4}$ $a_{4} \equiv a_{4}+\frac{2 \pi n}{L}$. The $a_{4}-a_{5}$ slice of the moduli space of the compactified theory is depicted in figure 3 , where we include all "images" along $a_{4}$ of the chosen vev.

Since $\mathrm{SU}(2) \rightarrow \mathrm{U}(1)$ by the expectation values (3.9), the theory has $x_{4}$-independent finite-action Euclidean monopole-instanton solutions, which are simply the dimensional reduction of the $4 \mathrm{~d}$ static 't Hooft-Polyakov monopole. Consider now the nonvanishing part of the action associated to such a $3 \mathrm{~d}$ monopole-instanton embedded in $\mathbb{S}^{1} \times \mathbb{R}^{3}$. Take the monopole solution to be $x_{4}$ independent and to have $A_{6}=0$, and $\left[A_{4}, A_{5}\right]=0$. By using steps similar to the Bogomolnyi's bound applied to dyons, ${ }^{5}$ we obtain, keeping only the nonvanishing terms in the bosonic action (2.2):

$$
\begin{aligned}
S & =\frac{1}{g_{4}^{2}} \int_{\mathbb{R}^{3} \times \mathbb{S}^{1}} \operatorname{tr}\left[\vec{B}^{2}+\left(\vec{D} A_{4}\right)^{2}+\left(\vec{D} A_{5}\right)^{2}\right] \\
& =\frac{L}{g_{4}^{2}} \int_{\mathbb{R}^{3}} \operatorname{tr}\left[\left(\vec{D} A_{4}-\sin \alpha \vec{B}\right)^{2}+2 \sin \alpha \vec{D} A_{4} \vec{B}+\left(\vec{D} A_{5}-\cos \alpha \vec{B}\right)^{2}+2 \cos \alpha \vec{D} A_{5} \vec{B}\right] \\
& \geq \frac{L}{g_{4}^{2}} \int_{\mathbb{R}^{3}} \operatorname{tr}\left[2 \sin \alpha \vec{D} A_{4} \vec{B}+2 \cos \alpha \vec{D} A_{5} \vec{B}\right] \\
& =\frac{L}{g_{4}^{2}} \int_{\mathbb{R}^{3}} \vec{\partial} \operatorname{tr}\left[2 \sin \alpha A_{4} \vec{B}+2 \cos \alpha A_{5} \vec{B}\right] \\
& =\frac{L}{g_{4}^{2}}\left[\sin \alpha a_{4}+\cos \alpha a_{5}\right] \int_{S_{\infty}^{2}} d \vec{\Sigma} \cdot \vec{B}^{3} \\
& =\frac{L}{g_{4}^{2}} \sqrt{\left(a_{5}^{2}+a_{4}^{2}\right)}(4 \pi) .
\end{aligned}
$$

\footnotetext{
${ }^{4}$ One way one can think of this is that $a_{4}$ always enters as $\partial_{4}+i a_{4}$ in the Lagrangian and thus can be shifted by $\frac{2 \pi}{L}$ by relabeling the Kaluza-Klein modes on the circle (or, equivalently, by a "large" gauge transformation).

${ }^{5}$ Earlier, when deriving the dyon equations (3.3), in the energy functional $E=\frac{1}{g_{4}^{2}} \int_{\mathbb{R}^{3}} \operatorname{tr}\left[\vec{B}^{2}+\vec{E}^{2}+\left(\vec{D} A_{5}\right)^{2}\right]$, we split the $\left(\vec{D} A_{5}\right)^{2}$ term such that it compensates both the electric and magnetic field. This yields the first order dyon equations given in (3.3); see ref. [8] for a review. On $\mathbb{R}^{3} \times \mathbb{S}^{1}$, since $A_{4}$ and $A_{5}$ are on different footing (compact vs. noncompact) and cannot be rotated to each other, the $\vec{B}^{2}$ term in the action should now split to compensate the two types of scalar terms, as in (3.10), when applying Bogomolnyi's technique to our problem.
} 


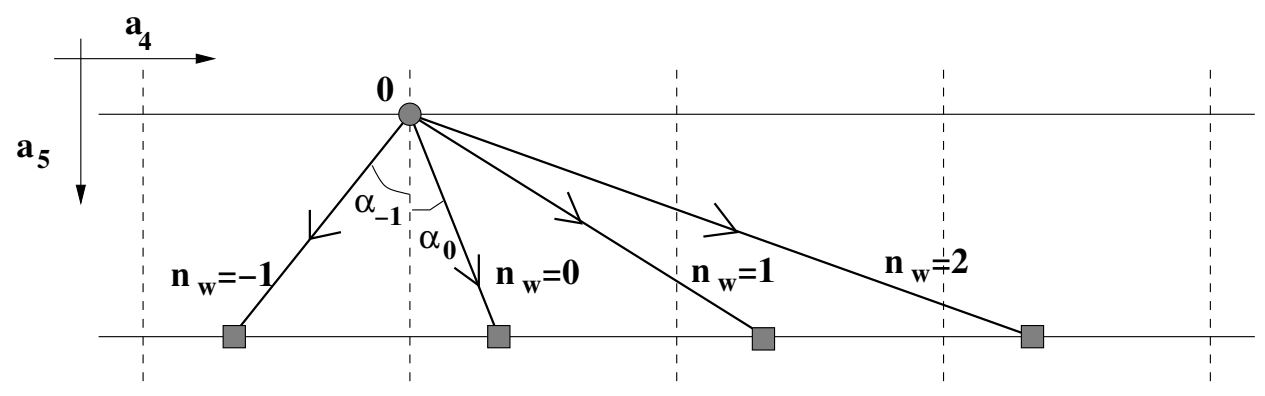

a) $n_{m}=+13 d$ instanton tower

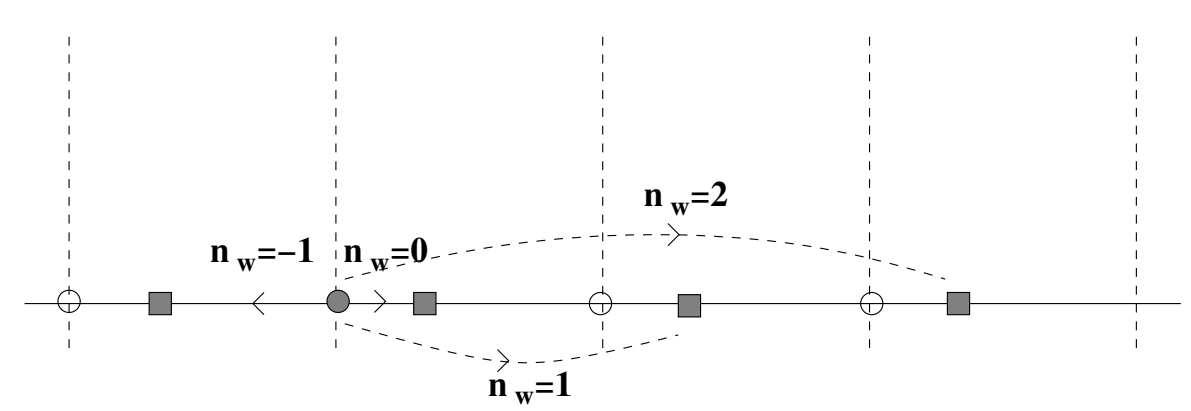

b) $n_{m}=+13 d$ instanton tower at $a_{5}=0$

Figure 3. Monopole-instanton solutions for $n_{m}=+1$ are represented by a line with an arrow pointing from the vev of $a_{4,5}$ at the center of the monopole, denoted by a circle, to the vev at infinity, denoted by a square (the vev at the center vanishes and is, on both pictures, taken to be the origin of coordinates in the $a_{4} / a_{5}$-plane). Since $a_{4}$ is an angular variable on $\mathbb{R}^{3} \times \mathbb{S}^{1}$, a tower of instanton-monopoles of "winding numbers" labelled by $n_{w}$ exists. The length of the arrow equals the distance between the vevs at the center of the monopole and infinity, $\sqrt{a_{5}^{2}+a_{4}\left(n_{w}\right)^{2}}$, and is proportional to the action of the corresponding topological defect. The $n_{m}=+1$ tower, shown in the upper figure, is composed of deformations (obtained by turning on $a_{5}$ ) of BPS monopoleinstantons and $\overline{\mathrm{KK}}$ twisted anti-instantons, shown in the lower figure (BPS monopoles have their arrows pointing to the right and $\overline{\mathrm{KK}}$-monopoles to the left). The $n_{m}=-1$ tower, not shown above, obtained by reverting all the arrows, is composed of deformations of $\overline{\mathrm{BPS}}$ anti-monopole-instantons and KK twisted monopole-instantons.

In the last step, we assumed that the magnetic charge is +1 . Furthermore, the last equality in (3.10) only holds when the r.h.s. is minimized with respect to $\alpha$ for the given value of the magnetic charge, i.e. $\alpha$ is given by the vevs (3.9) for the $A_{5}$ and $A_{4}$ fields as follows:

$$
e^{i \alpha}=\frac{a_{5}+i a_{4}}{\sqrt{a_{5}^{2}+a_{4}^{2}}} .
$$

This value of $\alpha$ is denoted by $\alpha_{0}$ in figure 3a. The action of the monopole is equal to the minimal value of the r.h.s. when the solution is BPS saturated:

$$
\begin{aligned}
& \vec{D} A_{4}-\sin \alpha \vec{B}=0, \\
& \vec{D} A_{5}-\cos \alpha \vec{B}=0 .
\end{aligned}
$$


Few comments are now in order:

1. For $\alpha=\pi / 2$, the first equation in (3.12) is the dimensional reduction of the usual instanton equation, $F=\tilde{F}$ (remembering that $D_{i} A_{4}=F_{i 4}$, etc.), now obeyed by a self-dual BPS-monopole instanton with $n_{m}=1$.

2. For $\alpha=0$, the second equation in (3.12) is the usual Bogomolnyi equation (see (3.3 with $\delta_{n_{e}}=0$ ) for a magnetic monopole particle with $n_{m}=1$ in macroscopically four dimensional space-time.

3. For $\alpha=-\pi / 2$, the first equation in (3.12) is the dimensional reduction of the antiinstanton equation, $F=-\tilde{F}$, which is obeyed by an anti-selfdual KK-monopoleinstanton with $n_{m}=1$ (recall that the KK-monopole instanton is self-dual and has $n_{m}=-1$, while the $\overline{\mathrm{BPS}}$-monopole-instanton is anti-selfdual and has $n_{m}=-1$ ).

So far, we have only addressed the $3 \mathrm{~d}$ BPS instanton embedded in $\mathbb{R}^{3} \times \mathbb{S}^{1}$ without winding number. ${ }^{6}$ We can generalize the above argument by incorporating the winding number $n_{w} \in \mathbb{Z}$. Recall that $a_{4}$ in (3.9) is really an angular variable on $\mathbb{R}^{3} \times \mathbb{S}^{1}$ and that $a_{4} \equiv a_{4} \bmod \frac{2 \pi}{L}$. Monopole-instanton solutions of higher action ("winding number") can be constructed allowing for larger separation between the scalar field eigenvalues at the center of the monopole and infinity - increasing in steps of $\frac{2 \pi n_{w}}{L}$ in the $a_{4}$ direction, as illustrated on figure 3. Then, repeating the steps in (3.10), we have for winding solutions saturating the BPS bound:

$$
\begin{aligned}
S_{n_{w}} & =\frac{L}{g_{4}^{2}}\left[\sin \alpha_{n_{w}}\left(a_{4}+\frac{2 \pi}{L} n_{w}\right)+\cos \alpha_{n_{w}} a_{5}\right] \int_{S_{\infty}^{2}} d \vec{\Sigma} \cdot \vec{B}^{3} \\
& =\frac{L}{g_{4}^{2}} \sqrt{a_{5}^{2}+\left(a_{4}+\frac{2 \pi}{L} n_{w}\right)^{2}}(4 \pi), \quad n_{w} \in \mathbb{Z}
\end{aligned}
$$

where:

$$
e^{i \alpha_{n_{w}}}=\frac{a_{5}+i\left(a_{4}+\frac{2 \pi}{L} n_{w}\right)}{\sqrt{a_{5}^{2}+\left(a_{4}+\frac{2 \pi}{L} n_{w}\right)^{2}}} .
$$

The BPS bound on the action (3.13) is achieved by solutions obeying: ${ }^{7}$

$$
\begin{aligned}
& \vec{D} A_{4}-\sin \alpha_{n_{w}} \vec{B}=0, \\
& \vec{D} A_{5}-\cos \alpha_{n_{w}} \vec{B}=0 .
\end{aligned}
$$

\footnotetext{
${ }^{6}$ The existence of the winding (also called "twisted", or "KK") monopole-instantons is only possible because the "Higgs field" $\sim e^{i L A_{4}}$ is compact. We note that the existence of extra monopole solutions in theories with compact Higgs fields has been noted, but not pursued, earlier, in the context of maximal abelian projection [9]. The advent of $D$-branes greatly helped the study of the twisted monopole-instantons, as they appear rather naturally in string theory brane constructions [10], see also [11].

${ }^{7}$ To avoid a possible confusion about the $x_{4}$ independence of the winding solutions, we note that all winding solutions are $x_{4}$ independent in a gauge where they asymptote to vacua with $a_{4}$ vevs differing by $2 \pi n_{w} / L$, while in a gauge where all solutions asymptote to a vacuum with fixed $a_{4}$, the winding solutions acquire $x_{4}$ dependence. The BPS bound is most simply derived in the first gauge.
} 
All the instantons in the tower have the same magnetic charge, but their topological charges differ by one unit. Interestingly, this tower is a deformation ${ }^{8}$ of the $a_{5}=0$ theory to nonzero $a_{5}$ and the instantons pertinent to this set-up arise from the self-dual 3d-instanton (BPS) for $n_{w} \geq 0$ and twisted ( $\left.\overline{\mathrm{KK}}\right)$ anti-instanton for $n_{w} \leq-1$. The existence of these two types of topological excitations, $3 \mathrm{~d}$ instantons and the twisted-instantons, is pertinent to the locally four-dimensional nature of the theory.

Since the action of a monopole-instanton that saturates the BPS bound (3.15) is determined by the vev at infinity, on figure 3 , it is geometrically represented by the length of the line $e^{9}$ stretching between the scalar eigenvalues at the origin and infinity. In (3.13), if we take $a_{5}=0$ and restrict attention to the $3 \mathrm{~d}$ instanton for which $n_{w}=0$, and take $a_{4}=\pi / L$, which corresponds to a center symmetric background, we obtain from (3.13), that the action of a $3 \mathrm{~d}$ instanton in a center-symmetric vacuum is $S_{n_{w}=0}=\frac{4 \pi^{2}}{g_{4}^{2}}$, which is half that of the $4 \mathrm{~d}$ instanton.

The $3 \mathrm{~d}$ monopole-instantons of magnetic charge +1 induce amplitudes proportional to $e^{-S_{n_{w}}} e^{i \sigma} \times$ (fermion zero modes) in the long distance effective Lagrangian, where $\sigma$ is the dual photon defined in (2.10). As explained above, there is an infinite tower of such instanton amplitudes contributing to the effective Lagrangian even for an instanton with a given magnetic charge.

Incorporating the shift due to the $a_{5}$ vev and winding in the action (3.13), and recalling from (2.11) that $a_{5}=v, L a_{4}=\omega$, we thus find that the sum of magnetic charge +1 instanton amplitudes in the small- $L$ domain is, schematically:

$$
\begin{aligned}
& \text { (Instanton sum at small- } L) \sim e^{i \sigma} F(\omega) \\
& \qquad F(\omega) \equiv \sum_{n_{w} \in \mathbb{Z}} e^{-S_{n_{w}}}=\sum_{n_{w} \in \mathbb{Z}} e^{-\frac{4 \pi}{g_{4}^{2}} \sqrt{(v L)^{2}+\left(\omega+2 \pi n_{w}\right)^{2}}},
\end{aligned}
$$

where $F(\omega)$ is the the sum of the fugacities (i.e., of the $e^{-S}$ prefactors of the instanton amplitude) and $e^{i \sigma}$ incorporates the long-distance Coulomb interaction between the monopole-instantons. In the regime where $a_{5} / a_{4} \gg 1$ or $(L v \gg 1)$, the action can be approximated by:

$$
S_{n_{w}} \approx \frac{4 \pi v L}{g_{4}^{2}}+\frac{1}{2} \frac{4 \pi}{L v g_{4}^{2}}\left(\omega+2 \pi n_{w}\right)^{2},
$$

leading to the asymptotic expression for the small- $L$ instanton sum:

$$
\text { (Instanton sum at small- } L) \sim e^{i \sigma} e^{-\frac{4 \pi v L}{g_{4}^{2}}} \sum_{n_{w} \in \mathbb{Z}} e^{-\frac{1}{2} \frac{4 \pi}{L v g_{4}^{2}}\left(\omega+2 \pi n_{w}\right)^{2}} \text {. }
$$

This small- $L$ instanton sum converges fast for $\frac{L v g_{4}^{2}}{4 \pi} \ll 1$, which implies $\frac{a_{5} g_{4}^{2}}{a_{4}} \ll 1$. The combination of this with the condition $\frac{a_{5}}{a_{4}} \gg 1$, used to obtain (3.17), gives:

$$
a_{4} \ll a_{5} \ll \frac{a_{4}}{g_{4}^{2}},
$$

\footnotetext{
${ }^{8}$ Similar solutions in nonsupersymmetric Yang-Mills-adjoint-Higgs theories are considered in [12].

${ }^{9}$ In the brane description of $\mathcal{N}=4 \mathrm{SYM}$ these are related to the worldlines of Euclidean D0branes $[13,14]$.
} 


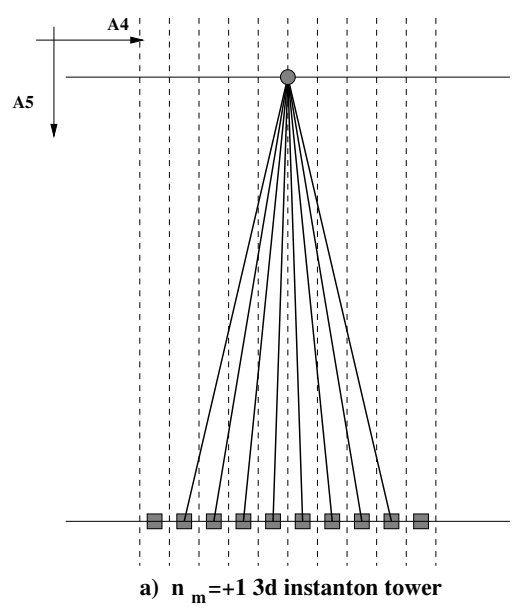

Figure 4. 3d-instantons in the magnetic charge +1 tower in the regime $a_{4} \ll a_{5} \ll a_{4} / g_{4}^{2}$. The tower is composed of deformation of BPS monopole instantons and $\overline{\mathrm{KK}}$ twisted anti-instantons. The properties of fermionic zero modes is dictated by the leading $a_{5}$ dependence for the low winding number instantons.

which can be accomplished at weak coupling. The monopole-instantons that contribute to the sum (3.18) are shown on figure 4.

In the next section, we will show that the small- $L$ and large- $L$ instanton sums (3.18) and (3.8) are, in fact, equivalent.

\subsection{3d-instanton/4d-dyon tower Poisson duality: first pass}

The statement of Poisson duality, which has been studied in $\mathcal{N}=4$ gauge theories in $[13,14]$ and in the $\mathcal{N}=2$ context in [15], is as follows: the small- $L$ (3.8) and large- $L$ (3.18) instanton sums, which, at first sight, look completely different, are in fact equivalent expressions, and one is the Poisson resummation ${ }^{10}$ of the other:

$$
\begin{aligned}
& e^{-\frac{4 \pi v L}{g_{4}^{2}}+i \sigma} \sum_{n_{w} \in \mathbb{Z}} e^{-\frac{1}{2} \frac{4 \pi}{L v g_{4}^{2}}\left(\omega+2 \pi n_{w}\right)^{2}} \times(\text { four-fermion operator }) \\
& =e^{-\frac{4 \pi v L}{g_{4}^{2}}+i \sigma} \sum_{n_{e} \in \mathbb{Z}} \sqrt{\frac{L v g_{4}^{2}}{8 \pi^{2}}} e^{-\frac{1}{2} \frac{v L g_{4}^{2}}{4 \pi} n_{e}^{2}+i n_{e} \omega} \times \text { (four-fermion operator) }
\end{aligned}
$$

Both of these are viewed as instanton sums: the first sum, (3.18), over the winding number incorporates 3d monopole-instantons and twisted (or winding) monopole-instantons and their Kaluza-Klein tower. The second sum, (3.8), which should perhaps be called a dyon sum, as its salient features are dictated by $4 \mathrm{~d}$ dyons, is a sum over the electric charges of the dyon tower. In this case, the instantons at large- $L$ are realized through dyon particles whose worldlines wrap around the large circle. As discussed earlier, the large- $L$ series converges fast for $L v g_{4}^{2} \gg 1$ and the small- $L$ series converges fast for $L v g_{4}^{2} \ll 1$.

The origin of the Poisson duality (3.20) can be simply understood as follows. In the regime when the expansions (3.2) and (3.18) make sense, i.e., at weak coupling and when

\footnotetext{
${ }^{10}$ See section 3.5 , where we prove a more general relation, which implies $(3.20)$.
} 
$L v \gg 1$, one can interpret the first and second sums in (3.20) in terms of the semiclassical expansion around a classical $x_{4}$ independent BPS monopole-instanton solution of zero winding/zero electric charge, respectively, of action $\frac{4 \pi v L}{g_{4}^{2}}$. The $4 \mathrm{~d}$ static monopole solution has a zero mode associated with global $\mathrm{U}(1)_{e}$ transformations, as already mentioned after (3.2). Let the corresponding collective coordinate be $\phi \in[0,2 \pi]$, a compact variable. It is useful to first recall that when quantizing static monopoles as particles on $\mathbb{R}^{3,1}, \phi$ is taken to be a function of time (by the coupling of internal and charge symmetries, corresponding to a time-dependent rotation of the monopole), and has a Lagrangian:

$$
\mathcal{L}_{\phi}=\frac{I}{2} \dot{\phi}^{2}
$$

where $I$ is the "moment of inertia" of the monopole of mass $M$ and "size" $R, I=M$ " $R^{2}$ " $=$ $\frac{4 \pi v}{g_{4}^{2}} \frac{1}{v^{2}}=\frac{4 \pi}{g_{4}^{2}}$. This is the Lagrangian of a particle on a circle of unit radius. The corresponding Hamiltonian is:

$$
H_{\phi}=\frac{1}{2 I} p_{\phi}^{2},
$$

where the eigenvalues of angular momentum $p_{\phi}$ are $n_{e}=0, \pm 1, \pm 2, \ldots$, the electric charges of the dyons (recall that the radius of the circle is unity).

When the path integral on $\mathbb{R}^{3} \times \mathbb{S}^{1}$ is considered, the "static" $x_{4}$-independent solutions become $3 \mathrm{~d}$ instanton-monopoles, and after changing variables to collective coordinates in the path integral, we have to integrate over all functions $\phi\left(x_{4}\right)$, corresponding to the classical paths the particle on a circle can take in a periodic time direction. The net result is that the path integral over the collective coordinate $\phi$ is equal to the partition function of the particle on a circle with Lagrangian $\mathcal{L}_{\phi}(3.21)$ at temperature $\frac{1}{L}$.

It is well known that there are two equivalent ways to compute the partition function $Z(L)=\operatorname{tr} e^{-L H_{\phi}}$ : one can either compute it in the operator formalism as a sum over states in the Hilbert space of $H_{\phi}$, or use the Feynman path integral to represent $Z(L)$ as a sum over classical paths of all possible winding numbers. Let us ignore the $A_{4}$ background momentarily. If one computes the partition function using the sum over eigenvalues of (3.22), one obtains from tre $e^{-L H_{\phi}}$ the sum over the electric charges of the dyon tower, the second line in (3.20). On the other hand, if the partition function is represented as a sum over winding classical paths labelled by winding number $n_{w}, \phi_{n_{w}}\left(x_{4}\right)=\frac{2 \pi n_{w}}{L} x_{4}+\phi_{0}\left(x_{4}\right)$, where $\phi_{0}$ is periodic, one ends up with the small- $L$ representation from the first line in (3.20). In other words, the two representations of $Z(L)$ are:

$$
\begin{aligned}
Z(L)=\operatorname{tr} e^{-L H_{\phi}} & \equiv \sum_{n_{e} \in \operatorname{Spec}\left(H_{\phi}\right)}\left\langle n_{e}\left|e^{-L H_{\phi}}\right| n_{e}\right\rangle=\sum_{n_{e} \in \operatorname{Spec}\left(H_{\phi}\right)} e^{-\frac{L n_{e}^{2}}{2 I}} \\
& \equiv \int_{\phi(0)=\phi(L)(\bmod 2 \pi)} D \phi(\tau) e^{-\int_{0}^{L} d \tau \frac{I}{2} \dot{\phi}^{2}} \\
& =\sum_{n_{w} \in \pi_{1}\left(S^{1}\right)} \sqrt{\frac{2 \pi I}{L}} e^{-\frac{I}{2 L}\left(2 \pi n_{w}\right)^{2}}
\end{aligned}
$$


where the prefactor on the second line in (3.20) is related to the properly normalized path integral over the periodic $\phi_{0}$. Since $\frac{1}{I}=\frac{g_{4}^{2} v}{4 \pi}$, we immediately see the equivalence of (3.23) to $(3.20)$.

The coupling to $A_{4}=\omega / L$ can be similarly understood by considering the static monopole in an external $A_{0}$ field, which modifies (3.21), (3.22), to $\mathcal{L}_{\phi}=\frac{I}{2}\left(\dot{\phi}+i A_{0}\right)^{2}$ and $H=\frac{1}{2 I} p_{\phi}^{2}-i A_{0} p_{\phi}$, respectively, and a subsequent continuation to Euclidean space. From the thermal analogy, it is also clear that at low temperature (large- $L$ ), the partition function converges faster if given as a sum over the quantized eigenvalues of $H$, while at high temperature (small- $L$ ) it is better represented as a sum over classical paths.

Going back to the monopole-instanton sums (3.20), the Poisson duality means that we can describe the system most conveniently in terms of the few leading topological defects in the respective sums wherever they converge fast. However, this is an issue of convenience and the physical content of the two sums is identical. Nevertheless, we would have to do much work to describe the small- $L$ phenomena by using the large- $L$ degrees of freedom and vice versa. The Poisson resummation also instructs us that the electric charge and winding number are dual variables. As is clear from the above discussion, in this system, the quantization of the dyon electric charge may also be thought as the dual of the quantization of winding number of $3 \mathrm{~d}$ monopole instantons. ${ }^{11}$

In the presence of massless fermions, the instanton amplitudes are associated with a number of fermionic zero modes. In general, the fermionic zero modes rotate as a function of the two adjoint Higgs vevs, $a_{4}$ and $a_{5}$, and vary throughout the moduli space. We postpone the detailed discussion of the preserved supersymmetries and the fermion zero mode structure to appendix A for conciseness. ${ }^{12}$ For now, we only note that in the limit that we derived the Poisson duality, $\alpha_{n_{w}} \approx 0$ for a large number of instantons in the $3 \mathrm{~d}$ tower (see figure 4 ); similarly in the semi-classical and non-relativistic limit of dyons, giving rise to the dense band of states shown in figure 2 , we have $\delta_{n_{e}} \approx 0$. In these two regimes, the wave functions of the fermion zero modes of the two towers are identical to leading order, as follows from eqs. (A.10) and (A.13). This demonstrates that the Poisson duality (3.20) is not harmed by the inclusion of fermionic zero modes.

\subsection{General Poisson duality, or $4 \mathrm{~d}$ dyon spectrum from $3 \mathrm{~d}$ instanton sums}

In this subsection, we demonstrate that the relativistic version of the BPS spectrum (3.1) and the central charge formula $Z_{\left(n_{m}, n_{e}\right)}$ for the magnetic dyon tower in $4 \mathrm{~d}$ can be extracted from the sum over the tower of $3 \mathrm{~d}$ instantons. This procedure is parallel to the well-known textbook example on the extraction of the energy spectrum of $\mathrm{O}(N)$ rigid rotator from the path integral formulation of quantum mechanics. See, for example, ref. [16]. We present the calculation in detail for pedagogical reasons.

Consider first the following small- $L$ sum over the instanton tower (3.16) labeled by the winding number $n_{w}$ and magnetic charge $n_{m}=1$. The winding number $n_{w} \in \mathbb{Z}$ is

\footnotetext{
${ }^{11}$ In the string theory embeddings this follows from momentum/winding number $T$-duality along $\mathbb{S}^{1}[13,14]$.

${ }^{12}$ However, this is an integral part of the discussion. The reader who is not familiar with this line of reasoning should study the derivations in appendix A.
} 
the integer part of the topological charge of the corresponding monopole-instanton in the tower. The sum (3.16) is a generalized fugacity $F(\omega)$ :

$$
F(\omega)=\sum_{n_{w} \in \mathbb{Z}} e^{-S_{n_{w}}}=\sum_{n_{w} \in \mathbb{Z}} e^{-\frac{4 \pi}{g_{4}^{2}} \sqrt{(v L)^{2}+\left(\omega+2 \pi n_{w}\right)^{2}}} .
$$

This is clearly a periodic function of holonomy $\omega, F(\omega+2 \pi)=F(\omega)$. Introduce its Fourier transform:

$$
F(\omega)=\sum_{n_{e} \in \mathbb{Z}} F_{n_{e}} e^{i \omega n_{e}},
$$

where $e^{i \omega n_{e}}$ is the canonical coupling of the background gauge field $A_{4}$ to electric charge $n_{e}, e^{i \int_{\mathbb{S}^{1}} n_{e} A_{4}} \equiv e^{i \omega n_{e}}$. Remembering that the fugacity we are calculating appears in the combination $e^{i \sigma} F(\omega)$, we deduce that the Fourier coefficients $F_{n_{e}}$ are associated with dyons of magnetic and electric charges $\left(1, n_{e}\right) . F_{n_{e}}$ should have an interpretation as the Boltzman weight, just like it was the case in our non-relativistic discussion in section 3.4, but now for a relativistic particle with Hamiltonian $H_{\phi}=\sqrt{M_{(1,0)}^{2}+v^{2} p_{\phi}^{2}}$ and eigenspectrum $M_{\left(1, n_{e}\right)}=$ $v \sqrt{\left(\frac{4 \pi}{g_{4}^{2}}\right)^{2}+n_{e}^{2}}$. We will indeed see that $F_{n_{e}} \sim e^{-L M_{\left(1, n_{e}\right)}}$. To this end, let us invert (3.25):

$$
\begin{aligned}
F_{n_{e}} & =\int_{0}^{2 \pi} \frac{d \omega}{2 \pi} F(\omega) e^{-i \omega n_{e}} \\
& =\sum_{n_{w} \in \mathbb{Z}} \int_{0}^{2 \pi} \frac{d \omega}{2 \pi} e^{-\frac{4 \pi}{g_{4}^{2}} \sqrt{(v L)^{2}+\left(\omega+2 \pi n_{w}\right)^{2}}} e^{-i \omega n_{e}} \\
& =\int_{-\infty}^{\infty} \frac{d \omega}{2 \pi} e^{-\frac{4 \pi}{g_{4}^{2}} \sqrt{(v L)^{2}+\omega^{2}}} e^{-i \omega n_{e}} .
\end{aligned}
$$

We will see below that $F_{n_{e}}$ equals the contribution of a single dyon pseudoparticle of electric charge $n_{e}$. Thus, the middle line of (3.26) expresses the single dyon contribution as a linear combination of contributions of an infinite number of winding monopole-instantons.

We now perform the integral in (3.26) by using the change of variables $\omega=v L \sinh t$, $d \omega=v L \cosh t d t:$

$$
F_{n_{e}}=\frac{v L}{4 \pi} \int_{-\infty}^{\infty} e^{-\frac{v L}{2}\left(\left(\frac{4 \pi}{g_{4}^{2}}+i n_{e}\right) e^{t}+\left(\frac{4 \pi}{g_{4}^{2}}-i n_{e}\right) e^{-t}\right)}\left(e^{t}+e^{-t}\right) d t,
$$

and then split the integrand in two. In the first one, we use $e^{t}=u$ and in the second $e^{-t}=u$. Then, we have $F_{n_{e}}=\frac{v L}{4 \pi}\left(F_{1 n_{e}}+F_{2 n_{e}}\right)$, where $F_{2 n_{e}}=F_{1 n_{e}}^{*}$, and obtain:

$$
\begin{aligned}
F_{1 n_{e}} & =\int_{0}^{\infty} e^{-\frac{v L}{2}\left(\left(\frac{4 \pi}{g_{4}^{2}}+i n_{e}\right) u+\left(\frac{4 \pi}{g_{4}^{2}}-i n_{e}\right) \frac{1}{u}\right)} d u=2 \sqrt{\frac{\left(\frac{4 \pi}{g_{4}^{2}}-i n_{e}\right)}{\left(\frac{4 \pi}{g_{4}^{2}}+i n_{e}\right)}} K_{1}\left(v L \sqrt{\left(\frac{4 \pi}{g_{4}^{2}}\right)^{2}+\left(n_{e}\right)^{2}}\right) \\
& =2 e^{-i \delta_{n_{e}}} K_{1}\left(L M\left(1, n_{e}\right)\right),
\end{aligned}
$$

where $K_{1}$ is the Bessel function, $M\left(1, n_{e}\right)$ is the mass of the dyon with electric charge $n_{e}$, and $\delta_{n_{e}}$ is the angle defined in finding the dyon equations of motions (3.3). Thus, $F_{n_{e}}$ 
becomes:

$$
F_{n_{e}}=\frac{v L}{4 \pi}\left(2 e^{-i \delta_{n_{e}}}+2 e^{i \delta_{n_{e}}}\right) K_{1}\left(L M\left(1, n_{e}\right)\right)=\frac{v L}{\pi} \cos \delta_{n_{e}} K_{1}\left(S\left(1, n_{e}\right)\right),
$$

where $S\left(1, n_{e}\right)$ is the action of the dyon (3.5).

Substituting (3.29) back into (3.25), the generalized Poisson duality is:

$$
\begin{aligned}
\sum_{n_{w} \in \mathbb{Z}} e^{-\frac{4 \pi}{g_{4}^{2}} \sqrt{(v L)^{2}+\left(\omega+2 \pi n_{w}\right)^{2}}} & =\sum_{n_{e} \in \mathbb{Z}} \frac{v L}{\pi} \cos \delta_{n_{e}} K_{1}\left(L M\left(1, n_{e}\right)\right) e^{i \omega n_{e}} \\
& =\sum_{n_{e} \in \mathbb{Z}} \frac{v L}{\pi} \cos \delta_{n_{e}} \sqrt{\frac{\pi}{2 L M\left(1, n_{e}\right)}} e^{-L M\left(1, n_{e}\right)} e^{i \omega n_{e}}
\end{aligned}
$$

In the second line, we used the $x \rightarrow \infty$ asymptotic expansion $K_{1}(x) \approx \sqrt{\frac{\pi}{2 x}} e^{-x}$. As promised, the sum over the $3 \mathrm{~d}$ instanton tower is Poisson dual to the sum over the spectrum of the $4 \mathrm{~d}$ dyon particle tower. The $4 \mathrm{~d}$ spectrum of dyons can easily be obtained through the formula:

$$
M\left(1, n_{e}\right)=-\lim _{L \rightarrow \infty}\left(\frac{1}{L} \log F_{n_{e}}\right) .
$$

This formula generalizes to dyons with higher magnetic charges as well. We note that in (3.31), the 1.h.s. is a $4 \mathrm{~d}$ quantity while the r.h.s. is given as an infinite sum over $3 \mathrm{~d}$ contributions comprising $F_{n_{e}}$, see (3.26), given in terms of sum over paths. ${ }^{13}$

In the semi-classical domain discussed in section 3.4, we assumed $\cos \delta_{n_{e}} \approx 1$ for the low-lying dyon band, and used the non-relativistic approximation for the dyon mass of eq. (3.2) and the expansion (3.17) for the twisted instanton action. Inserting these expansions, on both sides, we obtain exactly (3.20) of section 3.4; the prefactors also match exactly.

\subsection{Digression: the meaning of the sum}

One might ask what quantities in the $\mathcal{N}=2$ theory does the sum in the general Poisson duality formula (3.30) relate? In fact, this question was already addressed in [15]. It turns out that the r.h.s. of (3.30) is proportional to the large- $L$ dyon tower contribution to the Kähler potential, where the complex moduli $v=\left(A_{5}+i A_{6}\right) / \sqrt{2}$ and $\sigma-i b=\sigma-i \frac{4 \pi \omega}{g_{4}^{2}}$, recall (2.11), are used to parameterize the hyper-Kähler manifold. More explicitly, the

\footnotetext{
${ }^{13}$ This is actually a special and particularly simple case of a more general Poisson duality formula, formulated semi-classically by Gutzwiller and later generalized to an exact relation by Selberg [17]. The winding number is associated with the first homotopy group and the lengths of closed geodesics are in one-toone correspondence with the homotopy classes in the compact space. The electric charges provide spectral data about the Hamiltonian, which describes the $\mathrm{U}(1)_{e}$ collective coordinate of the monopole particle. Gutzwiller trace formula is a relation between the lengths of closed geodesics and the quantum spectrum of a Hamiltonian, and applies also to spaces with non-abelian isometry groups. It may be interesting to examine if there is anything of significance in this relation for the monopole physics and whether it has connections to a non-abelian generalization of T-duality in string theory.
} 
contributions of the dyon tower to the Kähler potential are given by: ${ }^{14}$

$$
K_{\text {dyon }}=\frac{1}{\sqrt{2} \pi^{\frac{3}{2}} L^{\frac{3}{2}}|v|^{\frac{1}{2}}} \sum_{n_{m}= \pm 1} \sum_{n_{e} \in \mathbb{Z}} \frac{e^{-L|v| \sqrt{\left(\frac{4 \pi}{g_{4}^{2}}\right)^{2}+n_{e}^{2}+i \omega n_{e}+i \sigma n_{m}}}}{\left[\left(\frac{4 \pi}{g_{4}^{2}}\right)^{2}+n_{e}^{2}\right]^{\frac{1}{4}}},
$$

while the Poisson resummed Kähler potential, expressed now as a sum over the contributions of winding solutions, and thus appropriate at small $L$, is:

$$
K_{\text {winding }}=K_{\text {dyon }}=\frac{1}{\pi L^{2}|v|} \sum_{n_{m}= \pm 1} \sum_{n_{w} \in \mathbb{Z}} e^{-\frac{4 \pi L}{g_{4}^{2}} \sqrt{|v|^{2}+\left(\frac{\omega+2 \pi n_{w}}{L}\right)^{2}}+i \sigma n_{m}} .
$$

Note that $K_{\text {winding }}=K_{\text {dyon }}$ is precisely equivalent our equation (3.30) in the $g_{4}^{2} \rightarrow 0$ limit (i.e., with $\cos \delta_{n_{e}} \approx 1$ ).

The sum (3.32) over the dyon tower contributions to the Kähler potential was obtained in [15] by solving the equations [18] obeyed by the hyper-Kähler metric on the moduli space of the $\mathcal{N}=2$ theory on $\mathbb{R}^{3} \times \mathbb{S}^{1}$ iteratively for weak coupling. Thus, the expressions for the Kähler potentials (3.32) and (3.33) are valid in the limit $|v| \gg \Lambda_{\mathcal{N}=2}$, but for arbitrary values of $|v| L$. Note that in the regime where (3.32), (3.33) are valid, $|v| \Lambda_{\mathcal{N}=2} \gg 1$, there is no "wall-crossing" and that this regime is different from the large- $L$ (but arbitrary coupling) regime $|v| L \gg 1$ studied in [18]. We also note that in [15], instead of the Kähler potential (3.33) for $v$ and $\sigma-i b$, the Kähler metric component $g_{v v^{*}}$ was actually given, but it is a simple matter to check their equivalence.

We see that the Poisson duality explained in section 3.4 is actually more generally valid. However, it is difficult to give a semiclassical test of this more general duality, since the fermion zero modes of the different winding and dyon backgrounds are different (see appendix A) and demonstrating the Poisson duality would entail showing that the sums over the various four-fermion operators agree (ref. [15] only provided a semiclassical test of the duality in the simplifying regime where the four-fermion operators of the two towers are identical, just as we did near eq. (3.20)). However, the derivation starting from the Kähler potential or Kähler metric [15], which assumes the validity of the "wall-crossing" formula [18], shows that the duality is clearly valid - the four-fermion terms follow from the metric and the relevant zero modes appearing in the four-fermion amplitude adjust themselves as the moduli and quantum numbers of the relevant instantons vary.

To summarize, in sections 3.4, 3.5, and 3.6, we argued that in the semiclassical regime along the path $C$ of figure 1, the topological defects responsible for confinement at small and large $L$ are related by Poisson duality. At large $L$, the $\left(n_{m}, n_{e}\right)=(1,0)$ and $(1,1)$ members of the dyon tower and their antiparticles survive continuation to the strong-coupling $u \sim 1$ domain and become massless at the monopole and dyon points, respectively. At small $L$, it is the lowest action winding monopole-instantons, $\left(n_{m}, n_{w}\right)=(1,0)$ and $(-1,-1)$ and their anti-instantons that play a crucial role in the dynamics of confinement. Next, we discuss these confinement mechanisms in turn.

\footnotetext{
${ }^{14} \mathrm{Up}$ to an overall constant, which is the same in (3.32) and (3.33).
} 


\section{Mass deformed Seiberg-Witten theory on $\mathbb{S}^{1} \times \mathbb{R}^{3}$}

In the next section 4.1, we recall the description of the monopole and dyon condensation in the mass-perturbed theory at large $L, L \gg \Lambda_{\mathcal{N}=2}^{-1}$. The local dynamics at distances smaller than $L$ is the one of $4 \mathrm{~d} \mathrm{SW}$-theory. If we are interested in physics at distances larger than the $\mathbb{S}^{1}$ size $L$, then the dynamics needs to be described by the dimensional reduction of the relevant SQED. We give a description based on various $3 \mathrm{~d}$ infrared dualities. ${ }^{15} \mathrm{In}$ section 4.2, we elucidate the role of the monopole-instantons and magnetic bions in the less well-known small- $S^{1}$ confinement and chiral symmetry breaking dynamics.

\subsection{The large- $L$ regime}

On $\mathbb{R}^{4}$, Seiberg and Witten (SW) showed that the theory around any one of the two singular points in moduli space can be described as $d=4, \mathcal{N}=2$ SQED. These points are usually referred to as monopole $(u=1)$ and dyon $(u=-1)$ points. Near these points, the $(1,0)$ and $(1,1)$ dyons, respectively, are described by hypermultiplets charged under the electromagnetism of the relevant dual photon multiplet, $\mathcal{A}_{\mathcal{D}}$; we note again that there is no globally valid effective field theory that describes both the monopole and dyon point in moduli space. Precisely at the monopole/dyon points, these hypermultiplets become massless.

When a mass perturbation reducing the $\mathcal{N}=2$ supersymmetry to $\mathcal{N}=1$ is added, SW theory exhibits confinement due to the condensation of the monopoles or dyons. Explicitly, using $\mathcal{N}=1$ superfields, the superpotential of SQED near either of the two singular points has the form:

$$
W=\sqrt{2} A_{D} Q \widetilde{Q}+m u\left(A_{D}\right)
$$

where $Q$ and $\widetilde{Q}$ are chiral monopole multiplets with charges, \pm 1 , respectively under the electromagnetism of the dual photon vector multiplet, $\mathcal{A}_{D} . A_{D}$ is the $\mathcal{N}=1$ scalar component of $\mathcal{A}_{D}$ and the first term in (4.1) is required by $\mathcal{N}=2$ supersymmetry of the $m=0$ theory. Near the monopole point,

$$
A_{D} \approx c(u-1)
$$

The vacuum of the theory can be found by solving simultaneously the $D$ - and $F$-term equations. Vanishing of the dual $\mathrm{U}(1) D$-term gives $D=Q^{\dagger} Q-\widetilde{Q}^{\dagger} \widetilde{Q}=0$. The critical point of the superpotential is $\frac{\partial W}{\partial u}=\frac{\partial W}{\partial Q}=\frac{\partial W}{\partial \widetilde{Q}}=0$ and yields:

$$
Q \widetilde{Q}=-\frac{m}{c \sqrt{2}}, \quad c(u-1) \widetilde{Q}=0, \quad c(u-1) Q=0 .
$$

This means that monopoles, which are massless particles in the $\mathcal{N}=2$ theory, become tachyonic once the mass perturbation $m \ll \Lambda_{\mathcal{N}=2}$ is added. The mass-perturbed SW

\footnotetext{
${ }^{15}$ Section 4.1 uses a $4 \mathrm{~d} \mathcal{N}=1$ (or, equivalently, $3 \mathrm{~d} \mathcal{N}=2$ ) formalism for the compactified theory. The reasoning entering the description of the asymptotically long-distance physics is based on symmetries and known 3d dualities. A good background material for this part is found in the lecture notes [20]. Our description will be brief since it does not have significant overlap with the rest of our discussion.
} 
theory exhibits a magnetic Higgs mechanism, has a mass gap and two isolated vacua (corresponding to either the monopole or dyon points). Confinement of electric charges is due to the Abrikosov-Nielesen-Olesen strings of the dual abelian Higgs model (SQED) describing the long-distance dynamics at the monopole or dyon point. ${ }^{16}$ Restoring explicit factors of $\Lambda_{\mathcal{N}=2}$, the monopole (dyon) condensate is $\langle Q\rangle \sim \sqrt{m \Lambda_{\mathcal{N}=2}}$ and the mass gap $M^{2}$ (i.e., the masses of the dual photon vectormultiplet and the monopole hypermultiplet) and the confining string tension $\Sigma$ are given by:

$$
M^{2} \sim e_{D}^{2} m \Lambda_{\mathcal{N}=2}, \quad \Sigma \sim m \Lambda_{\mathcal{N}=2}
$$

where $e_{D}$ is the IR-free dual photon coupling:

$$
e_{D}^{2} \sim \frac{16 \pi^{2}}{\log \frac{\Lambda_{\mathcal{N}=2}}{m}},
$$

which, in the mass-perturbed theory, is "frozen" due to $\langle Q\rangle \neq 0$.

As already mentioned in the Introduction, while the effective SQED descriptions of the monopole and dyon points are valid at $L \Lambda_{\mathcal{N}=2} \gg 1$, we do not know how continue them to the small- $L$ regime. First, recall that the monopole/dyon point SQEDs are effective theories, valid at energies below $\Lambda_{\mathcal{N}=2}$, and upon compactification, once $L \Lambda_{\mathcal{N}=2}$ becomes of order unity, the effective theory description breaks down since the IR free dual photon coupling (4.5) becomes strong at the scale $1 / L$. Second, as will become clear from the small- $L$ description of the dynamics in section 4.2 , for $0<L \Lambda_{\mathcal{N}=2} \ll 1$, there is a globally valid weak-coupling description of the two vacua of the mass-perturbed SW theory, while such a global description is lacking at large $L$. Thus, as $L$ is decreased, the two mutually nonlocal descriptions have to merge into a single one and it is beyond our current knowledge to describe this in field theory.

A more modest task is to consider a compactification of the SW theory on $\mathbb{R}^{3} \times \mathbb{S}^{1}$ of a radius such that $L \gg 1 / \Lambda_{\mathcal{N}=2}$. Then, the local dynamics is essentially four dimensional and the SQED descriptions near the monopole or dyon points in moduli space considered above are certainly valid. If we are interested in physics at distances larger than the $\mathbb{S}^{1}$ size $L$, we can describe the dynamics by the dimensional reduction of the relevant SQED. For this purpose, SW theory near the monopole/dyon points reduces to $\mathcal{N}=4 \mathrm{SQED}$ in $3 \mathrm{~d}$ - the dimensional reduction of the $4 \mathrm{~d}$ dual photon SQED with superpotential (4.1); for now, we ignore the mass perturbation. The $3 \mathrm{~d}$ gauge coupling at the UV cutoff of the $3 \mathrm{~d}$ theory is $e_{3}^{2}=e_{D}^{2}(L) / L$, where $e_{D}^{2}$ is the $4 \mathrm{~d}$ dual photon coupling.

A convenient way to study the $\mathcal{N}=43 \mathrm{~d}$ SQED is to start with $\mathcal{N}=23 \mathrm{~d}$ SQED. By adding a gauge-singlet chiral multiplet $\Psi$ with a superpotential $W=\sqrt{2} \Psi Q \widetilde{Q}$ (and a normalization of the kinetic term appropriate to an $\mathcal{N}=4$ multiplet), one obtains $\mathcal{N}=4$ SQED in 3d. As we will see shortly, this way of realizing the $\mathcal{N}=4$ SQED in $3 \mathrm{~d}$ is useful, because it will allow us to explore the IR dynamics through a chain of known $3 \mathrm{~d}$ dualities; see [20] for a review. This is also necessary, since the dimensional reduction of the $4 \mathrm{~d}$ IR free SQED with massless charged fields is not IR free and exhibits nontrivial dynamics.

\footnotetext{
${ }^{16}$ See [19] for detailed calculations for an $\mathrm{SU}(N)$ gauge group.
} 
Let us start with the $\mathcal{N}=23 \mathrm{~d}$ SQED as there is a duality relating it to a global (nongauge) theory. The vacuum of the $\mathcal{N}=23 \mathrm{~d}$ SQED theory is one complex dimensional and is parameterized, asymptotically, by three chiral superfields $\left\{V_{+}, V_{-}, M\right\}$; the first two $V_{+} \sim e^{\Phi^{D}}=e^{\phi^{D}+i \sigma^{D}}, V_{-} \sim e^{-\Phi^{D}}$ labeling the Coulomb branch (which splits in two ${ }^{17}$ ), and a meson superfield $M=Q \widetilde{Q}$ denoting the Higgs branch. Ref. [21] argues that the three branches meet at the origin of the moduli space and they are in fact related by a triality exchange symmetry $V_{+} \rightarrow V_{-} \rightarrow M \rightarrow V_{+}$. Ref. [21] also provides evidence that $\mathcal{N}=2$ SQED in $3 \mathrm{~d}$ is dual to a Wess-Zumino (WZ) model with superpotential:

$$
W=V_{+} V_{-} M
$$

in the infrared, ${ }^{18}$ where $V_{+}, V_{-}$, and $M$ are unconstrained chiral superfields.

Now, we can study $\mathcal{N}=43 \mathrm{~d}$ SQED by adding the image of the superpotential $W=$ $\sqrt{2} \Psi Q \widetilde{Q}$ in the $V_{+} V_{-} M$ WZ model. Since $Q \widetilde{Q}$ is the meson $M, W=\sqrt{2} \Psi Q \widetilde{Q} \equiv \Psi M$, and thus, the superpotential of the $V_{+} V_{-} M$ theory is deformed to:

$$
W=V_{+} V_{-} M+\Psi M
$$

The last term gives mass to $M$ and $\Psi$ and removes them from the effective theory. This leaves us with $V_{+}$and $V_{-}$with no superpotential. The IR-dual description of $3 \mathrm{~d} \mathcal{N}=4$ SQED is thus a free field theory. $V_{+}$and $V_{-}$parametrize a four-real dimensional manifold; this is a local patch of a hyper-Kähler manifold with quaternionic dimension one (presumably, the Atiyah-Hitchin manifold [2, 23]).

Finally, let us study the effect of the mass deformation in SW theory (adding this perturbation in the long-distance $3 \mathrm{~d}$ theory assumes that the mass gap (4.4) obeys $M \ll e_{3}^{2}$, since the IR-dual (4.7) is only valid at scales well below $e_{3}^{2}$; recall also that $e_{3}^{2} \ll 1 / L$ ). Based on our understanding of SW theory on $\mathbb{R}^{4}$, we expect that the mass-perturbed theory should have a mass gap. Indeed, the mass perturbation of superpotential (4.7) is:

$$
W=V_{+} V_{-} M+\Psi M+\epsilon \Psi
$$

with $\epsilon \sim \sqrt{m \Lambda_{\mathcal{N}=2}}$, whose critical points lead to a unique vacuum at $M=-\epsilon, V_{\mp}=0$, $\Psi=0$. It is easy to see that all excitations acquire mass and the theory is gapped, just as its $4 \mathrm{~d}$ counterpart.

These consistency checks are the most we can achieve using the $3 \mathrm{~d}$ language, since we are considering distances $\gg L$ and the $4 \mathrm{~d}$ language of massless monopoles becoming tachyonic and condensing is not the appropriate one here.

\footnotetext{
${ }^{17}$ Here, $\phi^{D}$ is the component of the $4 \mathrm{~d}$ dual photon gauge field along the compact direction and $\sigma^{D}$ is the $3 \mathrm{~d}$ scalar "dual photon" of the dimensionally reduced $4 \mathrm{~d}$ dual photon of SW theory (we do not see a way to avoid this potentially confusing terminology).

${ }^{18}$ With canonical $3 \mathrm{~d}$ normalization of the fields, the dimensionful coefficient in (4.6) should be $\sim e_{3}$ (although this is irrelevant for the long-distance dynamics of the WZ model, which is described by a 3d strongly-coupled "Wilson-Fisher" SCFT); the same factor makes up the dimensions in (4.7) as well.
} 


\subsection{The small- $L$ regime}

We now consider the limit of small circle size. In the small $\mathbb{S}^{1} \times \mathbb{R}^{3}$ domain of the $\mathcal{N}=2$ theory, there are two types of $3 \mathrm{~d}$ monopole instantons as well as their Kaluza-Klein towers, which were described in section 3.3. For definiteness, anticipating the applications to the mass-deformed theory, we will consider a point in moduli space where $a_{5}=0, a_{4} \neq 0$. Further, assuming $a_{4} \sim \pi / L \gg \Lambda_{\mathcal{N}=2}$, weak-coupling semiclassical methods are clearly applicable. In the region of moduli space chosen, the angle $\alpha$ of eq. (3.11) equals $\pm \pi / 2$ and the monopole instanton solutions are (anti-)self-dual, as explained after eq. (3.12). The action of the solutions of magnetic charge \pm 1 and arbitrary winding is given in (3.13).

The self-dual solutions are the BPS monopole-instanton $\left(n_{m}, n_{w}\right)=(1,0)$ of action $b=\frac{4 \pi}{g_{4}^{2}} L a_{4}=\frac{4 \pi}{g_{4}^{2}} \omega\left(\right.$ see (3.13) with $n_{w}=0,(2.11)$, and note that we take $\left.0 \leq \omega \leq 2 \pi\right)$ ). Below, we will denote the 't Hooft operator generated by the BPS monopole-instantons by $\mathcal{M}_{1}$. The other lowest action self-dual solution is the KK monopole and $\left(n_{m}, n_{w}\right)=$ $(-1,-1)$, which has action action $\frac{8 \pi^{2}}{g_{4}^{2}}-b=\frac{4 \pi}{g_{4}^{2}}(2 \pi-\omega)$ (see (3.13) with $\left.n_{w}=-1\right)$. The 't Hooft vertex associated with this instanton will be denoted by $\mathcal{M}_{2}$. Since we are at small $L$ here, it will be sufficient to consider only the contributions of these two lowest action solutions. These solutions have four fermion zero modes each (and, since they are self-dual, their zero modes are chiral in $4 \mathrm{~d}$ sense, see also appendix A). To summarize, the magnetic and topological charges $\left(Q_{m}, Q_{T}\right)$ associated with these instantons (and the corresponding anti-instantons) are:

$$
\begin{array}{ll}
\mathcal{M}_{1}:\left(+1,+\frac{1}{2}\right) & \mathcal{M}_{2}:\left(-1,+\frac{1}{2}\right) \\
\overline{\mathcal{M}}_{1}:\left(-1,-\frac{1}{2}\right) & \overline{\mathcal{M}}_{2}:\left(-1,-\frac{1}{2}\right) .
\end{array}
$$

Note that the topological charge equals $\frac{1}{2}$ only at the center-symmetric point $\omega=\pi$.

These monopole-instantons generate four-fermi interactions in the effective longdistance lagrangian, of the form:

$$
L_{4 \mathrm{~F}}=\mathcal{M}_{1}+\mathcal{M}_{2}+\overline{\mathcal{M}}_{1}+\overline{\mathcal{M}}_{2}
$$

where the amplitudes associated with these instanton events (and their anti-instantons) are given by:

$$
\begin{aligned}
& \mathcal{M}_{1}=e^{-b+i \sigma} \lambda \lambda \psi \psi \quad \mathcal{M}_{2}=\eta e^{+b-i \sigma} \lambda \lambda \psi \psi, \quad \eta \equiv e^{2 \pi i \tau}=e^{-\frac{8 \pi^{2}}{g_{4}^{2}}+i \theta} \\
& \overline{\mathcal{M}}_{1}=e^{-b-i \sigma} \bar{\lambda} \bar{\lambda} \bar{\psi} \bar{\psi} \quad \overline{\mathcal{M}}_{2}=\bar{\eta} e^{+b+i \sigma} \bar{\lambda} \bar{\lambda} \bar{\psi} \bar{\psi} .
\end{aligned}
$$

The combination $\mathcal{M}_{1} \mathcal{M}_{2}$ with eight zero mode insertions is the $4 \mathrm{~d}$-instanton amplitude and has the form $\eta(\lambda \lambda \psi \psi)^{2}$ given in (2.3). This amplitude, as noted in section 2.1, reduces the chiral $\mathrm{U}(1)_{R}$ symmetry down to $\mathbb{Z}_{8}$, which is a true anomaly-free symmetry of the quantum theory. Clearly, the chiral four-fermion operators in (4.11) flip sign under $\mathbb{Z}_{8}$. This implies that for the operators in (4.11) to remain invariant, one needs a discrete shift in the $\sigma$ field, of the form:

$$
\mathbb{Z}_{8}: \sigma \rightarrow \sigma+\pi
$$


The intertwining of the dual photon shift symmetry with continuous global symmetries, similar to our case of a discrete chiral symmetry (4.12), has been noted in [22]. Since (4.12) is not a continuous symmetry for $\sigma$, but just a $\mathbb{Z}_{2}$ shift symmetry, one may expect that an operator of the form $\cos (q \sigma), q=0(\bmod 2)$, may be induced. ${ }^{19}$ However, a bosonic potential is forbidden by the large amount of supersymmetry of the $\mathcal{N}=2$ theory, hence it is not generated. In other words, since the operators (4.11) in effective Lagrangian have more than two zero modes, the $\mathcal{N}=2$ theory on $\mathbb{S}^{1} \times \mathbb{R}^{3}$ cannot induce a superpotential and the moduli space is not lifted (in the $\mathcal{N}=2$ theory, the operators with four-fermion zero modes contribute to the hyper-Kähler metric). The IR physics is described as a threedimensional non-linear sigma model with target space $\mathcal{M}$, a hyper-Kähler manifold with quaternionic dimension one $[2,23]$. The IR field theory is described in terms of gapless bosonic degrees of freedom, $\phi, \phi^{\dagger}, b \pm i \sigma$, see (2.11), and their fermionic superpartners.

\subsection{1 $\mathcal{N}=1$ perturbation at small $\mathbb{S}^{1} \times \mathbb{R}^{3}$}

Adding a mass term for the chiral multiplet reduces the $\mathcal{N}=2$ supersymmetry to $\mathcal{N}=1$ and has the effect of lifting the $\psi$ zero modes from the instanton amplitudes (4.11). The mass perturbation (the kinetic term has an $\mathcal{N}=2$ normalization) is:

$$
\int d^{2} \theta m \operatorname{tr} \Phi^{2}+\int d^{2} \bar{\theta} m \operatorname{tr} \bar{\Phi}^{2}=m \operatorname{tr}(\bar{\psi} \bar{\psi}+\psi \psi)+\ldots
$$

Soaking up the $\psi$ zero modes from (4.11) with the mass perturbation induces the following 3d instanton amplitudes:

$$
\begin{array}{ll}
\mathcal{M}_{1}=m e^{-b+i \sigma} \lambda \lambda, & \mathcal{M}_{2}=m \eta e^{+b-i \sigma} \lambda \lambda, \\
\overline{\mathcal{M}}_{1}=m e^{-b-i \sigma} \bar{\lambda} \bar{\lambda}, & \overline{\mathcal{M}}_{2}=m \eta e^{+b+i \sigma} \bar{\lambda} \bar{\lambda}
\end{array}
$$

each of which carries only two zero modes and, as in (4.11), we use $\eta \equiv e^{2 \pi i \tau}=e^{-\frac{8 \pi^{2}}{g_{4}^{2}}+i \theta}$.

Four of the zero modes of the $4 \mathrm{~d}$ instanton are also lifted, as is transparent from the combination $\mathcal{M}_{1} \mathcal{M}_{2} \sim \eta(\lambda \lambda)^{2}$. Consequently, the axial symmetry is reduced to $\mathbb{Z}_{4}$. As in the $\mathcal{N}=2$ theory, the invariance of the amplitudes (4.14) demands that $\sigma \rightarrow \sigma+\pi$ when we apply a discrete chiral rotation to fermions. Since (4.14) carry just two zero modes, they now also generate a superpotential, given by:

$$
W_{\mathbb{R}^{3} \times \mathbb{S}^{1}} \sim m\left(e^{-\mathbf{B}}+\eta e^{\mathbf{B}}\right),
$$

\footnotetext{
${ }^{19}$ The BPS and KK instanton amplitudes, and the $4 \mathrm{~d}$ instanton 't Hooft vertices actually coincide in $\mathcal{N}=2 \mathrm{SYM}$ and the non-supersymmetric QCD(adj) with two massless Weyl adjoint fermion. Consequently, these two theories possess identical discrete and continuous chiral symmetries. On the other hand, the magnetic bion operator is generated only in SYM with $\mathcal{N}=1$ and in $\mathcal{N}=0$ QCD(adj). We expect that the microscopic reason behind the non-formation of the magnetic bions in $\mathcal{N}=2 \mathrm{SYM}$ is the existence of exactly massless adjoint Higgs scalars. It is desirable to show this explicitly, and we hope to address this question in future work. When the adjoint Higgs scalar is lifted by a soft mass term, the theory reduces to $\mathcal{N}=1 \mathrm{SYM}$, and a magnetic bion induced potential $\sim \cos 2 \sigma$ is both permitted and generated.
} 
where $\mathbf{B}$ is an $\mathcal{N}=1$ chiral superfield whose lowest component is $b-i \sigma$. Clearly, there are two isolated vacua located located at:

$$
\left\langle e^{\mathbf{B}}\right\rangle=\left\langle e^{b-i \sigma}\right\rangle= \pm \eta^{-\frac{1}{2}}= \pm e^{\frac{4 \pi^{2}}{g_{4}^{2}}-i \frac{\theta}{2}}, \quad \text { or } \quad\left\langle a_{4}\right\rangle=\frac{\pi}{L}, \quad\langle\sigma\rangle=\left(\frac{\theta}{2}, \frac{\theta}{2}+\pi\right) .
$$

Note that (4.16) implies that at $L \ll \Lambda_{\mathcal{N}=2}^{-1}$ the semiclassical reasoning is justified, as the vacuum is at the center-symmetric point $a_{4}=\frac{\pi}{L}$. These results are well known $[2,21,24]$.

Here, we would like to instead discuss the physics of the superpotential in some detail. Let us first study the bosonic potential, ${ }^{20}$ omitting the inessential overall constant and taking $\theta=0$ :

$$
V(b, \sigma) \sim\left|\frac{\partial W}{\partial \mathbf{B}}\right|^{2}=e^{-2 b}+\eta^{2} e^{2 b}-\eta\left(e^{-2 i \sigma}+e^{2 i \sigma}\right),
$$

which, when expanded around center-symmetric vacuum $\langle b\rangle=\frac{4 \pi^{2}}{g_{4}^{2}}$, takes the form:

$$
V(\langle b\rangle+b, \sigma) \sim \eta\left(e^{-2 b}+e^{2 b}-e^{-2 i \sigma}-e^{2 i \sigma}\right) \sim \eta \cosh 2 b-\eta \cos 2 \sigma .
$$

Evidently, in the effective lagrangian, the mass gap for gauge fluctuations (recall that $\sigma$ is the dual photon) is generated by the operator $e^{ \pm 2 i \sigma}$, and for the spin-zero scalar fluctuation, it is generated by $e^{ \pm 2 b}$.

As discussed in ref. [3, 4], in gauge theories with massless adjoint fermions, the $3 \mathrm{~d}$ instanton and twisted instanton do not generate a mass gap for the gauge fluctuations due to their fermionic zero mode structure, dictated by the index theorem [6, 7]. Rather, the bosonic potential is induced by monopole-antimonopole pairs (multi-instanton amplitudes), which may be viewed as composites, with opposite chirality zero modes soaked-up. The bosonic potential is sourced by the amplitudes $\left[\mathcal{M}_{1} \overline{\mathcal{M}}_{1}\right],\left[\mathcal{M}_{2} \overline{\mathcal{M}}_{2}\right],\left[\mathcal{M}_{1} \overline{\mathcal{M}}_{2}\right]$, and $\left[\mathcal{M}_{2} \overline{\mathcal{M}}_{1}\right]$, which are composites of (4.14). The magnetic and topological charges and the amplitudes associated with these instanton-antiinstanton events are:

$\begin{array}{cccc}\text { composite } & \left(Q_{m}, Q_{T}\right) & \text { amplitude } & \left(Q_{\text {dil }}, Q_{m}, Q_{T}\right) \\ {\left[\mathcal{M}_{1} \overline{\mathcal{M}}_{1}\right]} & (0,0) & e^{-2 b} & (-2,0,0) \\ {\left[\mathcal{M}_{2} \overline{\mathcal{M}}_{2}\right]} & (0,0) & e^{+2 b} & (+2,0,0) \\ {\left[\mathcal{M}_{1} \overline{\mathcal{M}}_{2}\right]} & (+2,0) & e^{+2 i \sigma} & (0,+2,0) \\ {\left[\mathcal{M}_{2} \overline{\mathcal{M}}_{1}\right]} & (-2,0) & e^{-2 i \sigma} & (0,-2,0)\end{array}$

where the quantum numbers of the individual instantons are given in (4.14). The last column and $Q_{\text {dil }}$, a pseudo-quantum number, will be explained in section 4.2.2.

Eq. (4.19) listing the origin of the various terms in the scalar potential (4.17) is quite interesting. Note that all $\left[\mathcal{M}_{i} \overline{\mathcal{M}}_{j}\right]$ events have vanishing topological charge, i.e., they are indistinguishable from the perturbative vacuum in that sense. However, the $\left[\mathcal{M}_{1} \overline{\mathcal{M}}_{2}\right]$ (and

\footnotetext{
${ }^{20}$ The kinetic terms of the fields $\sigma$ and $b$ are $\frac{1}{2} \frac{g_{4}^{2}}{(4 \pi)^{2} L}\left[\left(\partial_{i} b\right)^{2}+\left(\partial_{i} \sigma\right)^{2}\right]$, corresponding to a Kähler potential $K=\frac{g_{4}^{2}}{2(4 \pi)^{2} L} \mathbf{B}^{\dagger} \mathbf{B}$.
} 
its anti-molecule) events carry two units of magnetic charge. Thus, they can be distinguished from the vacuum and have been called "magnetic bions" in [3, 4]. They provide an example of stable semiclassically calculable bound states of a monopole-instanton and a twisted monopole anti-instanton. The contribution of the magnetic bion amplitude to the effective Lagrangian, adapting the results of $[4,26]$ to the mass-deformed $\mathcal{N}=2$ theory, is:

$$
\begin{aligned}
{\left[\mathcal{M}_{1} \overline{\mathcal{M}}_{2}\right] } & \sim \mathcal{A} e^{-2 S_{0}} e^{2 i \sigma}, \quad \text { where } \\
\mathcal{A} & \sim \int_{0}^{\infty} d r e^{-V_{\text {eff },} \mathcal{M}_{1} \overline{\mathcal{M}}_{2}(r)}=\int_{0}^{\infty} d r e^{-\left(2 \times \frac{4 \pi L}{g_{4}^{2} r}+\left(4 n_{f}-2\right) \log r\right)}, \quad n_{f}=1 .
\end{aligned}
$$

The physics behind the "effective potential" $V_{\text {eff }}$ is that the "magnetic" (due to exchange of $\sigma$ ) and "electric" (due to exchange of $b$-scalar) repulsive interactions between the constituents of the bion ${ }^{21}$ are balanced by fermion zero-mode exchange induced attraction (the choice $n_{f}=1$ reflects the presence of a single massless Weyl adjoint flavor in the massperturbed $\mathcal{N}=2$ theory). This results in stable topological molecules of size $\ell_{\text {bion }} \sim L / g_{4}^{2}$ (see [26] for details) and a well behaved integral dominated around $r \sim \ell_{\text {bion }}$. The integral in the second line of (4.20) is over the radial separation $|\mathbf{r}|=r$ between $\mathcal{M}_{1}$ and $\overline{\mathcal{M}}_{2}$ monopole-instantons in the Euclidean setting, and is an example what is called a quasizero mode (the center of mass position of the $\left[\mathcal{M}_{1} \overline{\mathcal{M}}_{2}\right]$ molecule is an exact zero mode and the Gaussian fluctuations around these molecules are small). A correct treatment of the instanton-anti-instanton requires care due to these (quasi)-zero modes, and doing so yields a result in exact agreement with supersymmetry. In section 4.2 .2 , we will identify a new type of topological molecule, which is more subtle to identify, but plays a useful role in center-symmetry realization.

It is important to note that the existence of these non (anti)self-dual topological excitations transcends supersymmetry: while studying only the superpotential (4.15) and inferring the resulting potential (4.17) correctly incorporates their effect by the familiar "power of supersymmetry", it hides the physics of balancing repulsive and attractive forces, which also holds in nonsupersymmetric theories with multiple adjoint fermions.

Thus, we learn that in $\mathcal{N}=2 \mathrm{SYM}$ theory softly broken down to $\mathcal{N}=1$, at small- $L$, the mass for the dual photon is induced by composite topological excitations, the "magnetic bions" $\left[\mathcal{M}_{1} \overline{\mathcal{M}}_{2}\right]{ }^{22}$ Clearly, this is an exotic generalization of Polyakov's confinement mechanism to a locally four dimensional gauge theory. In a straightforward generalization of Polyakov's mechanism, the mass gap would occur due to operators $e^{-S_{0}} e^{ \pm i \sigma}$, typical for monopole-instantons without any fermion zero modes. This operator is forbidden in our theory due to the discrete chiral symmetry (4.12), but the operator $e^{2 i \sigma}$ is allowed and generated by magnetic bions.

It is well known that the generation of mass gap for the dual photon $\sigma$ also implies confinement of electric charge [5]. The mass gap for gauge fluctuations (the mass, $M$, of $\sigma$

\footnotetext{
${ }^{21}$ The fact that the $b$-field is not gapped (classically and to all orders in perturbation theory) in the supersymmetric case accounts the factor of two in front of the $1 / r$ repulsion in $V_{\text {eff }}$ compared to refs. [4, 26].

${ }^{22}$ We note that the magnetic bion size in $\mathcal{N}=2$ broken down to $\mathcal{N}=1$ or $\mathcal{N}=0$ also depends on the mass of the adjoint Higgs scalar if the mass $m \lesssim L / g_{4}^{2}$. See section 6 for further discussion.
} 
and $b$ ) and the tension of the confining electric flux tube $\Sigma$ between two static sources can be semiclassically calculated:

$$
\begin{aligned}
M^{2} & \sim \frac{m^{2}}{g_{4}^{8}} e^{-\frac{8 \pi^{2}}{g_{4}^{2}}} \sim m^{2} \times\left(\Lambda_{\mathcal{N}=2} L\right)^{4}, \\
\Sigma & \sim \frac{g_{4}^{2}}{L} M \sim m \Lambda_{\mathcal{N}=2} \times\left(\Lambda_{\mathcal{N}=2} L\right),
\end{aligned}
$$

where the coupling is taken at the scale $L$ and subleading logarithmic dependence on $\Lambda_{\mathcal{N}=2} L$ is neglected in the last equality on each line. The mass gap $M$, including the $g_{4^{-}}$ dependent prefactor can be inferred from the recent calculation in multi-flavor $\mathrm{QCD}(\mathrm{adj})$ of [26], while the result for the string tension follows from [5]. ${ }^{23}$

Equations (4.21) for the mass gap and string tensions can be compared to the corresponding expressions for $\mathbb{R}^{4}$ given in (4.4). Clearly the mass gap $M^{2}$ in (4.21) and on $\mathbb{R}^{4}$ have different power dependence on $m$, while the string tensions $\Sigma$ at both large and small $L$ scale as $m \Lambda_{\mathcal{N}=2}$. The small- $L$ values of $\Sigma$ and $M$ both increase with $L$ at fixed $\Lambda_{\mathcal{N}=2}$ and presumably saturate to the $\mathbb{R}^{4}$ values near $\Lambda_{\mathcal{N}=2} L \sim 1$, the region where we lack control over the theory (the string tension $\Sigma$ can be defined at any $L$ from the area law obeyed by a Wilson loop of appropriate size $\in \mathbb{R}^{3}$ and $M$ from the exponential fall-off of the two-point function of the gauge invariant magnetic field strength $B^{k} \sim \epsilon^{k i j} \operatorname{tr}\left[F_{i j}\left(\Phi+\frac{1}{L} \Omega\right)\right]$, where $\Omega$ is the Wilson line around $\mathbb{S}^{1}$ ).

As opposed to magnetic bions, the other composites from (4.19), the $\left[\mathcal{M}_{i} \overline{\mathcal{M}}_{i}\right]$ amplitudes, responsible for generating a potential for $b$ (or $a_{4}$ ), do not even carry a magnetic charge! Yet, both types of topological defects play crucial role in the dynamics, including center-symmetry realization and confinement, as we now discuss.

\subsubsection{Center-symmetry realization and center-stabilizing bions}

The potential for the $b$-field, $\sim e^{-2 b}+\eta^{2} e^{2 b}$, from eq. (4.17) generates a non-perturbatively induced repulsive interaction between the eigenvalues of the Wilson line around $\mathbb{S}^{1}$. The minimum (4.16) is at $b=\frac{4 \pi^{2}}{g_{4}^{2}}$, or, in terms of the Wilson line, it is:

$$
\Omega=e^{i A_{4} L}=\left(\begin{array}{c}
e^{i \frac{\pi}{2}} \\
\\
e^{-i \frac{\pi}{2}}
\end{array}\right), \quad \operatorname{tr} \Omega=0,
$$

up to gauge rotations. This is the unique center-symmetric vacuum of the theory on $\mathbb{R}^{3} \times \mathbb{S}^{1}$. The origin of the center-stabilizing operator in the Lagrangian are the $\left[\mathcal{M}_{1} \overline{\mathcal{M}}_{1}\right]$ and $\left[\mathcal{M}_{2} \overline{\mathcal{M}}_{2}\right]$ induced amplitudes in $(4.19) .{ }^{24}$

The amplitude associated with $\left[\mathcal{M}_{1} \overline{\mathcal{M}}_{1}\right]$ generates the run-away potential $e^{-2 b}$ for the eigenvalues of the Wilson line and forces them to be as far apart as possible. This part

\footnotetext{
${ }^{23}$ The same $g_{4}^{2}$ scaling of the mass gap as in (4.21) is obtained if one follows the normalizations in the $\mathcal{N}=1$ supersymmetric calculation of $[25]$.

${ }^{24}$ The remarks here and in section 4.2 .3 below hold for the mass-deformed $\mathcal{N}=2$ theory as well as for the pure $\mathcal{N}=1$ super-Yang-Mills theory, with the appropriate scale matching $\Lambda_{\mathcal{N}=1}^{3}=m \Lambda_{\mathcal{N}=2}^{2}$ applied; for this reason, in eqs. (4.28), (4.30), and (4.31) below we omit the factors of $m$ (along with other dimensionful factors) in the superpotential.
} 
is similar to the lifting of Coulomb branch in $\mathcal{N}=2 \mathrm{SYM}$ on $\mathbb{R}^{3}$, where the quantum theory does not have a ground state (or it is pushed to $b=\infty$ ) [22]. However, our theory has two interrelated differences with respect to $\mathcal{N}=2 \mathrm{SYM}$ on $\mathbb{R}^{3}$. The classical moduli is compact and the theory has an extra set of topological molecules, $\left[\mathcal{M}_{2} \overline{\mathcal{M}}_{2}\right]$. Were it not for $\left[\mathcal{M}_{2} \overline{\mathcal{M}}_{2}\right]$, the two eigenvalues would end up at $\pi$, corresponding to a center broken Wilson line, $\Omega=-1$. However, quite symmetrically, $\left[\mathcal{M}_{2} \overline{\mathcal{M}}_{2}\right]$ generates a repulsion between the two eigenvalues of the Wilson line which prevents them from coinciding. Consequently, the combination of the two center-stabilizing bions is to yield the center-symmetric minimum (4.22).

Here, we give a brief description of how the $\left[\mathcal{M}_{1} \overline{\mathcal{M}}_{1}\right]$ and $\left[\mathcal{M}_{2} \overline{\mathcal{M}}_{2}\right]$ contributions arise. A detailed discussion of these type of topological molecules in both supersymmetric and non-supersymmetric gauge theories will appear in [29] and the implication for the thermal deconfinement phase transition will appear in [30]. The contribution of the $\left[\mathcal{M}_{i} \overline{\mathcal{M}}_{i}\right]$ amplitude to the effective theory is, naively,

$$
\begin{aligned}
{\left[\mathcal{M}_{1} \overline{\mathcal{M}}_{1}\right] } & \sim \mathcal{A} e^{-2 S_{0}} e^{ \pm 2 b}, \quad \text { where } \\
\mathcal{A} & \sim \int_{0}^{\infty} d r e^{-V_{\text {eff, }} \mathcal{M}_{1} \overline{\mathcal{M}}_{1}(r)}=\int_{0}^{\infty} d r e^{-\left(-2 \times \frac{4 \pi L}{g_{4}^{2} r}+\left(4 n_{f}-2\right) \log r\right)}, \quad n_{f}=1,
\end{aligned}
$$

where now the interaction between constituents is all attractive: "magnetic" (due to exchange of $\sigma$-scalar) and "electric" (due to exchange of $b$-scalar) attractions and the fermion induced attraction (again, we put $n_{f}=1$ for the case of interest). The integral is dominated by the small- $r$ domain, where not only (4.23) is incorrect, it is also hard to make sense of constituents as the interaction becomes large. This is in sharp contrast with the magnetic bion $[4,26]$.

In contrast with the magnetic bions, these instanton-anti-instanton "molecules" are difficult to exhibit semiclassically, as their constituents have only attractive interactions, and naively the natural tendency is for these objects to annihilate. However, the amplitude associated with $\left[\mathcal{M}_{i} \overline{\mathcal{M}}_{i}\right]$ is not proportional to identity operator, but rather to $e^{ \pm 2 b}$. This means that, although in the sense of magnetic and topological charge these defects are indistinguishable from the perturbative vacuum, since the product of the amplitudes $\left[\mathcal{M}_{i} \overline{\mathcal{M}}_{i}\right]$ cannot be contracted to the identity, it should perhaps be seen as carrying a pseudoquantum number to distinguish it from perturbative vacuum. In fact, the coupling of $b$ to monopole-instantons can be thought of as the coupling of a massless "dilaton" [31] (dilatation is a classical symmetry, only globally broken by compactification and locally by the expectation value of $b$ ) or as "electric" charge, if one thinks of the compact $x_{4}$ as Euclidean time. Since the adjoint Higgs field is asymptotically of the form $A_{4}=a_{4} T^{3}\left(1-\frac{1}{a_{4} r}+\ldots\right)$, we can define a "dilaton" charge (or flux) associated with it by using Gauss' law:

$$
Q_{\mathrm{dil}}=\int_{S_{\infty}^{2}} \vec{\nabla} A_{4} \cdot d \vec{S}
$$

Note that $\vec{\nabla} A_{4}$ is the dimensional reduction of the Euclidean electric field $\vec{E}$ and integral may be interpreted, in some loose sense, as electric flux. ${ }^{25}$

\footnotetext{
${ }^{25}$ However, this is rather confusing as it forces us to think of monopole-instantons on $\mathbb{R}^{3} \times S^{1}$ as having
} 
Therefore, a more pragmatic way to think of the quantum numbers for topological defects on $\mathbb{R}^{3} \times \mathbb{S}^{1}$ is to incorporate $Q_{\text {dil }}$ and generalize the doublet of quantum numbers to a triplet:

$$
\left(Q_{m}, Q_{T}\right) \longrightarrow\left(Q_{\mathrm{dil}}, Q_{m}, Q_{T}\right)
$$

In this language, the charges of $\mathcal{M}_{1}$ are $\left(-1,+1, \frac{1}{2}\right)$ and the charges of $\left[\mathcal{M}_{1} \overline{\mathcal{M}}_{1}\right]$ are $(-2,0,0)$, as also shown in the last column in (4.19). In this sense, $\left[\mathcal{M}_{1} \overline{\mathcal{M}}_{1}\right]$ molecule is now distinguishable from perturbative vacuum. Monopoles and anti-monopoles carry same "dilation" or "electric" charges, and opposite magnetic charges. Also note that the same dilaton charge objects attract, as opposed to the fact that same magnetic charge objects repel.

Finally, we note that supersymmetry demands that the operators induced by instantonanti-instanton molecules should be there and their coefficients are identical to the one of magnetic bion up to a sign, see (4.18). In fact, using analytic continuation in the coupling constant $[33,34]$ or in the path integration contour over the quasi-zero mode [35], such saddle points of the Euclidean path integral can be defined. In particular, in supersymmetric quantum mechanics [35] and even $\mathcal{N}=1$ supersymmetric QCD [36] these complex saddle point contributions to the Euclidean path integral seem to be unambiguously defined. Since they are required by supersymmetry, we suspect that this is also the case in the mass-perturbed $\mathcal{N}=2$ theory (or in pure $\mathcal{N}=1$ ) at small $L$, an issue which is discussed in more detail in $[29,30]$.

\subsubsection{Chiral symmetry and the topological disorder operator}

The potential for the dual photon field $\sigma$ in (4.17) is $-\cos 2 \sigma$, with two isolated minima (4.16) located (for $\theta=0$ ) at $\sigma=0$ and $\pi$. The two minima are related to each other by the exact $\mathbb{Z}_{4}$ chiral symmetry of the mass-deformed $\mathcal{N}=2$ theory. The potential for the dual photon is generated by magnetic bion "molecules", $\left[\mathcal{M}_{1} \overline{\mathcal{M}}_{2}\right]$, of magnetic charge \pm 2 , whose dynamical stability is semiclassically calculable, as already explained.

The order parameter for chiral symmetry in the small- $\mathbb{S}^{1}$ domain of mass deformed Seiberg-Witten theory (as well as in the pure $\mathcal{N}=1$ theory) is:

$$
\left\langle e^{i \sigma}\right\rangle= \pm 1 \text {. }
$$

Contrary to assertions in literature stating that the chiral symmetry is broken by a local fermion bi-linear $\langle\operatorname{tr} \lambda \lambda\rangle$ [24], chiral symmetry is in fact broken by the vacuum expectation value of the topological disorder operator (4.26). If one performs a small- $L$ monopoleinstanton calculation of the fermion bilinear expectation value in the full $\mathrm{SU}(2)$ gauge theory, $\langle\operatorname{tr} \lambda \lambda\rangle$, similar to [24] but incorporating the long-range interactions in the monopoleantimonopole (or more precisely, bion-antibion) plasma, one finds that it is related to the expectation value of the disorder operator (4.26):

$$
\langle\operatorname{tr} \lambda \lambda\rangle \sim\left\langle e^{i \sigma}\right\rangle \eta^{\frac{1}{2}}
$$

both real electric and magnetic charges and leads to possible confusion with magnetic dyon particles on $\mathbb{R}^{4}$ (which carry genuine electric and magnetic charges). In the literature, the latter terminology is (appropriately) used in thermal Yang-Mills theory, see ref. [32], but here we refer to $Q_{\text {dil }}$ as dilaton charge to prevent confusion, and refer to $\mathcal{M}_{1}$ as monopole-instanton. 
This shows that the source of chiral symmetry breaking is the magnetic-bion induced potential (4.17) for the dual photon. A further argument that (4.27) is correct is that it correctly reproduces the values for the gaugino condensate in the two vacua (4.16), while due to the omission of the $\left\langle e^{i \sigma}\right\rangle$ factor on the r.h.s., eq. (4.7) in [24] only gives a single value. $^{26}$

As usual, the spontaneous breaking of chiral symmetry generates fermion mass. Consider the fermion bilinear terms arising from the superpotential:

$$
\frac{\partial^{2} W}{\partial B^{2}} \lambda \lambda+\text { c.c. } \sim\left(e^{-b+i \sigma}+\eta e^{+b-i \sigma}\right) \lambda \lambda+\text { c.c. },
$$

and evaluate them at the center-symmetric vacuum (4.16), to find that the fermion mass breaking the $\mathbb{Z}_{4}$ chiral symmetry:

$$
\eta^{\frac{1}{2}}\left(\left\langle e^{i \sigma}\right\rangle+\left\langle e^{-i \sigma}\right\rangle\right) \lambda \lambda= \pm 2 \eta^{\frac{1}{2}} \lambda \lambda
$$

is also due to (4.26).

Finally, we can consider taking the small and infinite $L$ limits of the superpotential (4.15). In the $\mathbb{R}^{3}$ limit, keeping $g_{3}^{2}=g_{4}^{2} L$ fixed, we find that $\eta \rightarrow 0$ and the two vacua (4.16) run-away to infinity. The superpotential reduces to the well-known run-away superpotential:

$$
W_{\mathbb{R}^{3}} \sim e^{-\mathbf{B}} .
$$

In the $\mathbb{R}^{4}$ limit, we should integrate out the chiral superfield $e^{-\mathbf{B}}$, as it does not represent a valid infrared degree of freedom - this can be seen, e.g., from the fact that the $\sigma, b$ kinetic terms, see footnote 20 , vanish in the infinite- $L$ limit; see also [21]. In doing so, we obtain the four dimensional gaugino condensate superpotential:

$$
W_{\mathbb{R}^{4}} \sim \pm \eta^{1 / 2}
$$

corresponding to the two isolated vacua of the $4 \mathrm{~d}$ mass perturbed $\mathcal{N}=2$ or pure $\mathcal{N}=1$ theory.

\section{Phase diagram and abelian (non-'t Hooftian) large-N limits}

On $\mathbb{R}^{4}$, confinement in an $\mathrm{SU}(2)$ gauge theory with $\mathcal{N}=2$ supersymmetry softly broken down to $\mathcal{N}=1\left(m \ll \Lambda_{\mathcal{N}=2}\right)$ is a version of abelian confinement. By this we mean that the long-distance effective Lagrangian is an abelian $\mathrm{U}(1)$ gauge theory, despite the fact that the microscopic theory is a non-abelian $\mathrm{SU}(2)$. The confinement of the electric charges is due to magnetic monopole or dyon condensation [1].

On the other hand, in the limit $m \gg \Lambda_{\mathcal{N}=2}$, where the adjoint Higgs multiplet decouples and the theory reduces to pure $\mathcal{N}=1$ SYM theory, there exists no known description of the gauge dynamics on $\mathbb{R}^{4}$ where abelianization takes place. It is usually believed that

\footnotetext{
${ }^{26}$ The breaking of chiral discrete or continuous symmetries by the disorder operator expectation value is also a generic feature of nonsupersymmetric gauge theories with fermions on $\mathbb{R}^{3} \times \mathbb{S}^{1}[27,28]$.
} 


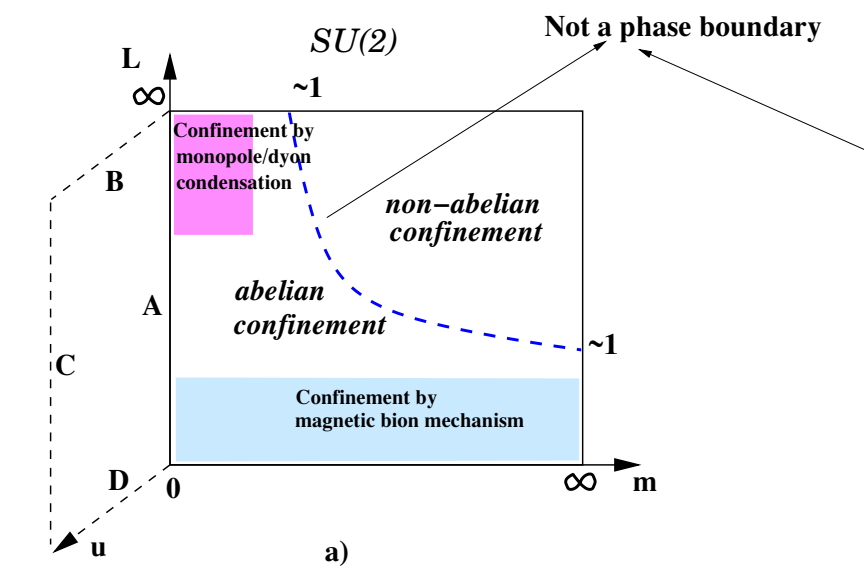

$S U(N)$, large $-N$

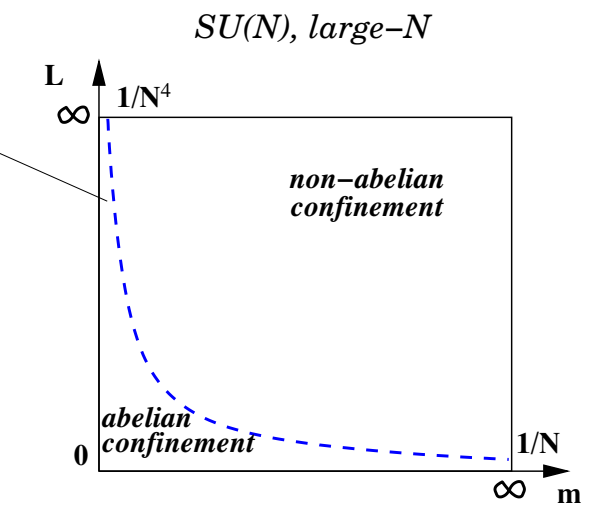

b)

Figure 5. The $\mathcal{N}=2$ theory broken down to $\mathcal{N}=1$ exhibits confinement. At small $m$ and/or small $L$ (in units of $\Lambda$ ) the dynamics abelianizes at large distances and the theory exhibits abelian confinement. The phase diagram in the $m-L$ plane for the small- $N$ theory is shown on the left figure, where the shaded areas indicate the calculable regimes at small and large $L$. The third $(u)$ direction - which allows to smoothly connect the topological excitations responsible for confinement at large and small $L$ via Poisson duality - is also indicated. At large- $N$, shown on the right figure, the calculable semi-classical confinement regime shrinks to a narrow sliver both in $m$ and $L$, in a correlated manner, as explained in the text.

there is no phase transition as the mass term is dialed from small to large and the theory moves from a regime of abelian confinement to non-abelian confinement.

In the pure $\mathcal{N}=1 \mathrm{SYM}$ theory, as well as in a large-class of non-supersymmetric gauge theories which remain center-symmetric upon compactification down to small radius, it has been recently understood that the $L \Lambda_{\mathcal{N}=1} \ll 1$ regime also exhibits abelian confinement. The confinement of the electric charges is now due to the magnetic bion mechanism $[3,4]$. Analogous to the mass-deformed theory, it is usually believed that there is no phase transition associated with center symmetry as the radius is dialed from small to large. At large- $L$, it is expected that non-abelian confinement should take place.

At what values of $m$ and $L$ does the metamorphosis from the abelian confinement (which we analytically understand) to the non-abelian confinement (which is not yet understood) take place? Naively, by dimensional analysis, one may argue that this should happen around $m \sim \Lambda_{\mathcal{N}=2}$ for the theory on $\mathbb{R}^{4}$ and at $L \sim \Lambda_{\mathcal{N}=1}^{-1}$ for the pure $\mathcal{N}=1$ SYM. Although this is true for $\mathrm{SU}(N)$ gauge theory with $N=2$, or a few, as shown in figure 5.a), it turns out to be incorrect especially at larger values of $N$.

This subtlety is associated with the regimes of gauge theory in which the long distance dynamics reduces to the one of the abelian subgroup $\mathrm{U}(1)^{N-1}$ and with the existence of light $W$-bosons whose masses scale as $\mathcal{O}(1 / N)$. This has been previously discussed by Douglas and Shenker in the mass deformed $\mathrm{SU}(N) \mathcal{N}=2$ supersymmetric Yang-Mills theory for $m$ scaling [19] and by Yaffe and one of us (M.Ü.) in the context of center symmetric vector-like gauge theories for $L$-scaling [37].

In this work, we argue that these two large- $N$ limits are indeed correlated in the phase diagram of the theory in $L-m$ plane. 
At large $\mathbb{S}^{1}$, in the $N \rightarrow \infty$ limit of the mass deformed theory, the Abelian long distance regime is preserved only if the mass deformation ${ }^{27} m$ is sent to zero as $m \sim \Lambda_{\mathcal{N}=2} / N^{4}$ (while the naive expectation would have been that $m \ll \Lambda_{\mathcal{N}=2}$ suffices). This follows from demanding the string tensions (see (4.4), which remains valid at large $N$ for the lowest string tensions) to be smaller than the lightest $W$ boson mass squared, $m_{W} \sim \Lambda_{\mathcal{N}=2} / N^{2}$ [19], a requirement which is crucial for the validity of the abelianized effective theory. Equivalently, the abelianized low energy effective theory is valid provided there exists a hierarchy of scales between the heaviest U(1)-photons and lightest W-bosons, which translates into:

$$
\frac{m_{\gamma}}{m_{W}} \sim \sqrt{\frac{m}{\Lambda_{\mathcal{N}=2}}} N^{2} \lesssim 1 \quad(L=\infty) .
$$

At small $\mathbb{S}^{1}$, on the other hand, it is the radius $L$ that has to be taken to zero in order for the abelianized description to be valid as $N \rightarrow \infty$. To see this, first take the large- $m$ limit with the appropriate scale matching $\Lambda_{\mathcal{N}=1}^{3}=m \Lambda_{\mathcal{N}=2}^{2}$. Then, we find that the abelian long distance regime is preserved only if the compactification radius $L$ is sent to zero as $L \sim 1 /\left(\Lambda_{\mathcal{N}=1} N\right)$. This follows from demanding that the mass of the lightest $W$ boson, $m_{W} \sim 1 /(L N)$ in the center-symmetric vacuum, be parametrically larger than the heaviest dual photon mass $m_{\gamma}$; we find:

$$
\frac{m_{\gamma}}{m_{W}} \sim\left(L \Lambda_{\mathcal{N}=1} N\right)^{3} \lesssim 1 \quad(m=\infty) .
$$

The conditions used in ref. [19] and ref. [37] for the validity of an abelianized low energy description are in fact equivalent. Thus, connecting these two large- $N$ limits, we conjecture that an abelian long distance regime only survives in a corner which becomes arbitrarily narrow as $N \rightarrow \infty$, as shown in figure 5.b). In particular, at any fixed non-zero $L \sim \mathrm{O}\left(N^{0}\right)$ and $m \sim \mathrm{O}\left(N^{0}\right)$, if one takes $N \rightarrow \infty$ first, there is no regime of the supersymmetric gauge theory in which the long distance dynamics remains abelian. At $L \sim \mathrm{O}\left(N^{0}\right)$ and $N \rightarrow \infty$, the theory exhibit volume independence, and behaves as if it is decompactified even when $L \Lambda_{\mathcal{N}=1} \ll 1$, see for example [37]. At any $m / \Lambda_{\mathcal{N}=2} \sim \mathrm{O}\left(N^{0}\right)$, as $N \rightarrow \infty$, we expect the adjoint scalar to completely decouple even when $m / \Lambda_{\mathcal{N}=2} \ll 1$, and the $\mathcal{N}=1$ dynamics to be independent of $m$. This mass independence is the equivalent of the large- $N$ volume independence in the non-abelian confinement domain. In other words, in the large- $N$ limit, we conjecture that all points in the non-abelian confinement domain in figure 5.b) are equivalent up to subleading corrections in $N$.

\section{Discussion and open problems}

By using field theory techniques and with rather minimal help from supersymmetry, we were able to answer the physical questions that we posed in the Introduction. For the $\mathcal{N}=1$ mass deformation of the $\mathcal{N}=2$ theory, we have shown that:

i) The theory confines and exhibits a mass gap through the magnetic bion mechanism. This mechanism also applies to a large class of non-supersymmetric theories, notably to QCD with multiple massless adjoint Weyl fermions.

\footnotetext{
${ }^{27}$ Note that at large- $N$ the mass deformation is $W=N m \operatorname{tr} \Phi^{2}[19]$.
} 
ii) The discrete chiral symmetry is broken due to the condensation of a disorder operator, and the $\mathrm{SU}(2)$ theory has two isolated vacua. In the effective long distance theory, this is the source of dynamical mass generation for fermions. The magnetic bions responsible for chiral symmetry breaking are the $\mathcal{M}_{1} \overline{\mathcal{M}}_{2}$ (3d BPS instantontwisted-antiinstanton) and $\mathcal{M}_{2} \overline{\mathcal{M}}_{1}$ (3d BPS anti-instanton-twisted instanton) topological molecules, generating the operators $e^{ \pm i 2 \sigma}$. These objects carry magnetic charge $Q_{m}= \pm 2$ and their stability can be semiclasically understood.

iii) The center symmetry is stabilized through non-perturbative $\mathcal{M}_{1} \overline{\mathcal{M}}_{1}$ (3d BPS instanton-antiinstanton) and $\mathcal{M}_{2} \overline{\mathcal{M}}_{2}$ (twisted instanton-antiinstanton) topological molecules generating the operators $e^{ \pm 2 b}$. These objects differ from the perturbative vacuum because they carry $Q_{\text {dil }}= \pm 2$ units of "dilatonic" charge.

iv) The Seiberg-Witten [1] and Polyakov [5] solutions on $\mathbb{R}^{4}$ and $\mathbb{R}^{3}$ have been known for almost two and over three decades, respectively. Recent progress in understanding confinement in gauge theories on $\mathbb{R}^{3} \times \mathbb{S}^{1}$, in particular the Polyakov-like magnetic bion mechanism in $\mathrm{QCD}(\operatorname{adj})$ and $\mathcal{N}=1 \mathrm{SYM}[3,4]$, permits us to demonstrate that the two type of confinements are in fact continuously connected. Namely, the topological excitations responsible for confinement at small- and large- $L$ : the winding monopole-instanton constituents of the magnetic bions and the dyon particles, respectively, are related by Poisson resummation.

There is a number of interesting questions that arise related to our construction:

1. Non-formation of magnetic bions in $\mathcal{N}=2$ theory. In the pure $\mathcal{N}=2$ theory, the global symmetries (except supersymmetry) are identical to those of nonsupersymmetric QCD with $n_{f}=2$ flavors of Weyl adjoint fermions. The latter theory, as well as the supersymmetric $\mathcal{N}=1$ theory, permit the magnetic bion induced operator $\sim \cos 2 \sigma$, but the theory with $\mathcal{N}=2$ supersymmetry does not. The fact that supersymmetry does not permit this operator is clear. Since leading topological defects have four zero modes, see (4.11), they do not induce a superpotential hence a bosonic potential is also not induced. However, while true as a symmetry argument, we think that it is interesting to understand, by microscopic means, the non-formation of magnetic bions in $\mathcal{N}=2$ SYM. We believe the non-formation of magnetic bions in $\mathcal{N}=2$ theory is due to the existence of exactly massless adjoint Higgs scalars counter-acting the multi-fermion induced attraction and that it would be of interest to show this explicitly.

2. The size of magnetic bions in softly broken $\mathcal{N}=2$ theory at small $m$. In the pure $\mathcal{N}=1$ theory, which is the $m=\infty$ limit of deformed SW theory with appropriate coupling matching, the typical magnetic bion size is - in a regime where we have semi-classical control over the dynamics $-\ell_{\text {bion }}(m=\infty) \sim L / g_{4}^{2}$. In the $\mathcal{N}=2$ theory with mass deformation, we expect the bion size to be dependent on $m$. If the correlation length for the scalar is smaller than the bion size, i.e., $m^{-1}<\ell_{\text {bion }}(\infty)$, the scalar decouples quickly and the bion size should remain as it is in the $\mathcal{N}=1$ theory. 
However, if $m^{-1}<\ell_{\text {bion }}(\infty)$, then we expect that the bion size should be proportional to an inverse power of $m$ and diverge in the $m=0$ limit. This is necessary for the non-formation of the bions in the pure $\mathcal{N}=2$ theory. A different behavior would invalidate the magnetic bion description of confinement in softly broken $\mathcal{N}=1$. Thus, it is desirable to study the adjoint mass dependence of the magnetic bion size.

3. Generalizations. It would be of interest to generalize our discussion to other gauge groups and consider the inclusion of other matter representations, where, possibly, new phenomena may be observed.

4. Topological molecules and the deconfinement transition. We have identified the topological molecules whose main role is to provide a center-symmetric vacuum. Similar and related molecules are also present in non-supersymmetric gauge theories, including pure Yang-Mills. These defects may play the pivotal role in the deconfinement phase transition, and may lead us towards a microscopic theory of deconfinement. Work in this direction is ongoing.

5. Relation to lattice studies of confinement. Let us finally comment on the nonsupersymmetric case. The bion confinement mechanism operative at small $L$ in the mass deformed $\mathcal{N}=2$ theory also applies to a large class of non-supersymmetric theories, notably to QCD(adj) theories with multiple massless adjoint Weyl fermions. This class of non-supersymmetric theories provides the first example where confinement and chiral symmetry breaking can be studied in a controlled manner in a locally $4 \mathrm{~d}$ theory.

It would be very interesting to know whether, in the non-supersymmetric multipleadjoint fermion "QCD-like" theories, a relation can be inferred between the confinement mechanism at small $L$ (which is reliably described by the magnetic bion mechanism) and large $L$ (where it is not understood). In the large- $L$ limit, lattice studies of pure Yang-Mills theory have shown the relevance of topological defects charged under the Abelian part (or the center) of the gauge group to the generation of mass gap and confinement (for a recent review, see [38]). Needless to say, this problem — addressing which would likely require both analytic and lattice input is left for future studies.

\section{Acknowledgments}

We thank P. Argyres, M. Shifman, A. Vainshtein, and A. Yung for useful discussions. This work was supported by the U.S. Department of Energy Grant DE-AC02-76SF00515 and by the National Science and Engineering Council of Canada (NSERC).

\section{A Chirality and fermion zero modes}

In the presence of massless fermions, the instanton amplitudes on $\mathbb{R}^{4}$ as well as the $3 \mathrm{~d}$ instantons (either at large or small $L$ ) acquire fermion zero modes. The number of zero 
modes is dictated by various index theorems, APS on $\mathbb{R}^{4}$, Callias on $\mathbb{R}^{3}$, and Nye-Singer on $\mathbb{R}^{3} \times \mathbb{S}^{1}$ interpolating between the two. In our discussion of Poisson duality, we have asserted certain conditions about the fermion zero modes and preserved supersymmetries in the background of topological defects. In particular, we will show that the duality described in (3.20) is unharmed by the inclusion of the four-fermi operators on both sides.

The issues about chirality and zero modes may be succinctly described starting with the supersymmetry transformation properties of a six dimensional theory, and then applying dimensional reduction in $x^{5,6}$ direction and compactification in $x^{4}$ direction. The notation is given in section 2.2 .

\section{A.1 4d-instanton}

As a warm-up, let us start with a $4 \mathrm{~d}$ instanton. The classical instanton configuration is the solution of self-duality condition $F_{\mu \nu}=\frac{1}{2} \epsilon_{\mu \nu \rho \sigma} F^{\rho \sigma}$ supplemented with vanishing fermionic fields. Since supersymmetry transformation relates variation of bosonic fields to fermions, the variation of bosonic fields are all zero, and the instanton background preserves the supersymmetries for which variation of fermionic field vanishes, i.e., $\delta \Psi=0$. The complement, which is not annihilated under supersymmetry transformation, gives the zero mode solution to the Dirac equation under the background of the instanton (or relevant topological excitation).

In the background of an instanton, setting the $A_{5}$ and $A_{6}$ scalars to zero, the supersymmetry variation of the fermion is $\left(\Gamma^{M N}=\left[\Gamma^{M}, \Gamma^{N}\right] / 2\right)$ :

$$
\begin{aligned}
\delta \Psi & =\left(\Gamma^{M N} F_{M N}\right) \varepsilon \\
& =\left(\Gamma^{\mu \nu} F_{\mu \nu}+2 \sum_{m=5,6} \Gamma^{\mu m} D_{\mu} A_{m}+i \sum_{m, n=5,6} \Gamma^{m n}\left[A_{m}, A_{n}\right]\right) \varepsilon \\
& =\left(\frac{1}{2} \Gamma^{\mu \nu} F_{\mu \nu}+\frac{1}{2} \Gamma^{\mu \nu}\left(\frac{1}{2} \epsilon_{\mu \nu \rho \sigma} F^{\rho \sigma}\right)\right) \varepsilon \\
& =\frac{1}{2} F_{\mu \nu}\left(\Gamma^{\mu \nu}+\frac{1}{2} \epsilon^{\mu \nu \rho \sigma} \Gamma_{\rho \sigma}\right) \varepsilon \\
& =\left[\frac{1}{2} F_{12}\left(\Gamma^{12}+\Gamma^{34}\right)+\frac{1}{2} F_{13}\left(\Gamma^{13}-\Gamma^{24}\right)+\ldots\right] \varepsilon,
\end{aligned}
$$

where we used the self-duality of the solution in the third line above. The final equality in (A.1) implies, for non-vanishing $F_{\mu \nu}$, that the supersymmetry preserved for a self-dual instanton is the one with parameter defined by the first line below, while a similar argument for the anti-self-dual instanton yields the supersymmetry defined by the second line:

$$
\begin{array}{ll}
\Gamma^{1234} \varepsilon=+\varepsilon & (4 \mathrm{~d} \text {-instanton background }) \\
\Gamma^{1234} \varepsilon=-\varepsilon & (4 \mathrm{~d} \text {-anti-instanton background }),
\end{array}
$$

with $\Gamma^{1234}=\Gamma^{1} \Gamma^{2} \Gamma^{3} \Gamma^{4}$. This defines a chirality condition for the zero mode structure of a $4 \mathrm{~d}$ instanton. We use a basis where the instanton zero modes are chiral and anti-instanton zero modes are anti-chiral. 


\section{A.2 3d monopole-instanton tower}

Now, consider the first order differential equations for the monopole-instantons that we derived in section 3.3 and figure out which half of the supersymmetries are preserved in this background for a given value of $\alpha$. The preserved supersymmetries can be found analogously, using $A_{6}=\left[A_{4}, A_{5}\right]=0$ and eq. (3.12) for the BPS monopole-instantons with $B_{i}=\frac{1}{2} \epsilon_{i j k} F_{j k}$ :

$$
\begin{aligned}
\delta \Psi & =\left(\Gamma^{M N} F_{M N}\right) \varepsilon \\
& =\left(\Gamma^{i j} F_{i j}+2 \sum_{m=4,5,6} \Gamma^{i m} D_{i} A_{m}+i \sum_{m=4,5,6} \Gamma^{m n}\left[A_{m}, A_{n}\right]\right) \varepsilon \\
& =\left(\Gamma^{i j} F_{i j}+2 \Gamma^{i 4} D_{i} A_{4}+2 \Gamma^{i 5} D_{i} A_{5}\right) \varepsilon \\
& =\left(\Gamma^{i j} F_{i j}+2 \Gamma^{i 4} \sin \alpha\left(\frac{1}{2} \epsilon_{i j k} F_{j k}\right)+2 \Gamma^{i 5} \cos \alpha\left(\frac{1}{2} \epsilon_{i j k} F_{j k}\right)\right) \varepsilon \\
& =F_{i j}\left(\Gamma^{i j}+\Gamma^{l 4} \sin \alpha \epsilon_{l i j}+\Gamma^{l 5} \cos \alpha \epsilon_{l i j}\right) \varepsilon \\
& =2\left[F_{12}\left(\Gamma^{12}+\Gamma^{34} \sin \alpha+\Gamma^{35} \cos \alpha\right)+\text { cyclic perm. }\{1 \rightarrow 2 \rightarrow 3\}\right] \varepsilon,
\end{aligned}
$$

leading to conserved supersymmetries defined through the equation:

$$
\left[\sin \alpha \Gamma^{1234}+\cos \alpha \Gamma^{1235}\right] \varepsilon=+\varepsilon,
$$

which is satisfied by half of the supersymmetries. Few comments, parallel to the bosonic discussion of section 3.3, are in order:

1. For $\alpha=\frac{\pi}{2}$, we have:

$$
\Gamma^{1234} \varepsilon=+\varepsilon \quad\left(\alpha=\frac{\pi}{2}, \text { self-dual BPS monopole-instanton }\right)
$$

Thus, $\varepsilon$ obeys the same "chirality" condition as the four dimensional instantons and the chirality of the $4 \mathrm{~d}$ instanton zero modes and the BPS monopole-instantons are aligned. These are the fermionic zero modes associated with the monopole-instanton when the vev is aligned purely in the $A_{4}$ direction.

2. For $\alpha=0$, we have:

$$
\Gamma^{1235} \varepsilon=+\varepsilon \quad(\alpha=0, \text { BPS monopole-instanton })
$$

This is also the condition satisfied for a monopole particle in $4 \mathrm{~d}$, as well as its lowlying dyons (since the bosonic background for $\alpha=0$ obeys the usual $4 \mathrm{~d}$ monopole particle BPS equation, see discussion after (3.12)). This condition will be particularly important when we consider Poisson-duality between the 4d-monopoles/dyons at large $\mathbb{S}^{1}$ and monopole instantons pertinent to small $\mathbb{S}^{1}$.

3. For $\alpha=-\pi / 2$, we have:

$$
\Gamma^{1234} \varepsilon=-\varepsilon \quad(\alpha=-\pi / 2, \overline{K K} \text { anti-instanton })
$$

In this case, $\varepsilon$ obeys the same "chirality" condition as the four dimensional antiinstantons. 
If we now consider the more general BPS and $\overline{\mathrm{KK}}$ monopole instantons whose action is given in (3.13), we need to generalize the equation obeyed by supersymmetries from (A.4) to:

$$
\left[\sin \alpha_{n_{w}} \Gamma^{1234}+\cos \alpha_{n_{w}} \Gamma^{1235}\right] \varepsilon_{n_{w}}=+\varepsilon_{n_{w}}
$$

For general values of $a_{4}$ and $a_{5}$, the supersymmetries respected by the various instantons with different winding number $n_{w} \in \mathbb{Z}$ are not the same. However, in the regime $a_{5} \gg a_{4}$, to leading order and for low-lying instantons in the tower, we can take:

$$
\sin \alpha_{n_{w}} \approx 0, \quad \cos \alpha_{n_{w}} \approx 1 .
$$

Hence, for the low-lying band of the instanton tower, we have:

$$
\Gamma^{1235} \varepsilon_{n_{w}}=+\varepsilon_{n_{w}}, \quad \text { independent of } n_{w} .
$$

The fermion zero-mode wave functions can be found by applying a broken supersymmetry transformation (i.e. the one orthogonal to (A.10)) to the monopole-instanton solution; we will not need the explicit form of these zero modes.

\section{A.3 Monopole-dyon particle tower}

In the background of a monopole or dyon particle, setting $A_{6}$ to zero and using the first order dyon differential equations (3.3) (thinking of $x^{4}$ as a compact Euclidean time direction, i.e., with $E_{i}=F_{4 i}$ ), we obtain the preserved supersymmetries:

$$
\begin{aligned}
\delta \Psi & =\left(\Gamma^{M N} F_{M N}\right) \varepsilon \\
& =\left(\Gamma^{\mu \nu} F_{\mu \nu}+2 \sum_{m=5,6} \Gamma^{\mu m} D_{\mu} A_{m}+2 i \Gamma^{56}\left[A_{5}, A_{6}\right]\right) \varepsilon \\
& =\left(\Gamma^{i j} F_{i j}+2 \Gamma^{4 i} F_{4 i}+2 \Gamma^{i 5} D_{i} A_{5}\right) \varepsilon \\
& =\left(\Gamma^{i j} \epsilon_{i j k} B_{k}+2 \Gamma^{4 i} E_{i}+2 \Gamma^{i 5} D_{i} A_{5}\right) \varepsilon \\
& =D_{i} A_{5}\left(\Gamma^{l m} \epsilon_{l m i} \cos \delta_{n_{e}}+2 \Gamma^{4 i} \sin \delta_{n_{e}}+2 \Gamma^{i 5}\right) \varepsilon
\end{aligned}
$$

For non-vanishing $D_{i} A_{5}$, the vanishing supersymmetry transformations can be found by multiplying the matrix equation in parenthesis with $\Gamma^{5 i}$ for a fixed $i$. They, as usual, yield, the same equation independent of the value of $i$, given by:

$$
\begin{aligned}
\left(\cos \delta_{n_{e}} \Gamma^{5 i l m} \epsilon_{l m i}-2 \sin \delta_{n_{e}} \Gamma^{54}+2\right) \varepsilon_{n_{e}} & =+\varepsilon_{n_{e}} \quad[i \text {-fixed }] \\
{\left[\cos \delta_{n_{e}} \Gamma^{1235}-\sin \delta_{n_{e}} \Gamma^{45}\right] \varepsilon_{n_{e}} } & =+\varepsilon_{n_{e}}
\end{aligned}
$$

For a generic dyon, the supersymmetries preserved are not aligned with the one of $3 \mathrm{~d}-$ instantons (A.8). However, in the limit when $\delta_{n_{e}} \sim 0$ :

$$
\Gamma^{1235} \varepsilon_{n_{e}}=+\varepsilon_{n_{e}}, \quad \text { independent of } n_{e},
$$

as in (A.10). The $\delta_{n_{e}} \sim 0$ condition, and hence (A.13) holds for the band of states for which $n_{e}^{2} \ll \frac{4 \pi^{2}}{g_{4}^{2}}$, see eq. (3.3). This guarantees the working of the approximate version Poisson duality (3.20) even when fermions are incorporated. Similar to the remarks after (A.10), the fermion zero modes can be found by applying a broken supersymmetry transformation to the bosonic solution. 


\section{B $\mathrm{SU}(N)$ generalization of Poisson duality}

We now turn to $\mathrm{SU}(N), N \geq 3$, generalization of the Poisson duality between the $4 \mathrm{~d}$ monopole/dyon tower and 3d instanton tower.

On $\mathbb{R}^{4}$, the set of vacua of the classical gauge theory is the space of commuting covariantly constant scalars $\left[\phi, \phi^{\dagger}\right]=0$, or $\left[A_{5}, A_{6}\right]=0$. For convenience, we take $A_{6}=0$ and $A_{5}=\operatorname{diag}\left[v_{1}, v_{2}, \ldots, v_{N}\right]$, and we choose a point in the moduli space where the long distance theory fully abelianizes, $\mathrm{SU}(N) \rightarrow \mathrm{U}(1)^{N-1}$. There are $N-1$ types of monopole particles whose charges are proportional to the $N-1$ simple roots $\alpha_{i}$ of the Lie algebra, of magnetic charges $Q_{i}=4 \pi \alpha_{i}, i=1, \ldots N-1$. The mass spectrum of these $N-1$ type of lightest monopole particles and their dyonic tower is:

$$
M_{i} \equiv M_{i, i+1}=\left|v_{i}-v_{i+1}\right| \sqrt{\left(\frac{4 \pi}{g_{4}^{2}}\right)^{2} n_{m}^{2}+n_{e}^{2}}
$$

For $n_{m}=0, n_{e}=1$, eq. (B.1) reduces to the mass formula for the lightest W-bosons due to adjoint Higgsing, $M_{i}=\left|v_{i}-v_{i+1}\right|$. For $n_{e}=0, n_{m}=1$, (B.1) is the semi-classical mass formula for the monopoles, $M_{i}=\left|v_{i}-v_{i+1}\right|\left(\frac{4 \pi}{g_{4}^{2}}\right)$. Our primary interest are the set of monopoles with $n_{m}=1$ and arbitrary electric charge $\sim n_{e} \alpha_{i}$.

For the theory compactified on $\mathbb{R}^{3} \times \mathbb{S}^{1}$, the Wilson line along the compact direction $\Omega=e^{i \int_{S^{1}} A_{4}}$ can also be interpreted as scalar from the lower dimensional point of view. However, $A_{4}$ differs from $A_{5}$ and $A_{6}$ since it is an angular variable, and as in our $\mathrm{SU}(2)$ example, this plays a crucial role. The vacua of the classical gauge theory on $\mathbb{R}^{3} \times \mathbb{S}^{1}$ are spanned by the space of commuting covariantly constant scalars:

$$
\left[A_{3+i}, A_{3+j}\right]=0, \quad i, j=1,2,3,
$$

which may be parameterized as three sets of $N$ eigenvalues,

$$
\begin{array}{ll}
A_{4}=\frac{1}{L} \operatorname{diag}\left(\omega_{1}, \omega_{2}, \cdots, \omega_{N}\right), & \sum_{i=1}^{N} \omega_{i}=0 \bmod 2 \pi, \\
A_{5}=\operatorname{diag}\left(v_{1}, v_{2}, \cdots, v_{N}\right), & \sum_{i=1}^{N} v_{i}=0 .
\end{array}
$$

For convenience, we set $A_{6}=0$. For generic configurations with distinct eigenvalues, the long distance theory fully abelianizes. At such points, the subgroup of global gauge transformations which preserve the diagonalized form (B.3) is the Weyl group $\mathcal{W}$ of $\mathrm{SU}(N)$, whose elements simultaneously permute the eigenvalues of the two scalars, $\left(L^{-1} \omega_{i}, v_{i}\right) \rightarrow$ $\left(L^{-1} \omega_{\sigma(i)}, v_{\sigma(i)}\right)$, where $\sigma \in \mathcal{S}_{N}\left(\mathcal{S}_{N}\right.$ is the $N$-element permutation group). In this sense, $\mathcal{S}_{N}$ acts on $N$ - "eigenbranes" whose positions are given by:

$$
\mathbf{r}_{i}=\left(L^{-1} \omega_{i}, v_{i}\right)
$$

as $\mathbf{r}_{i} \rightarrow \mathbf{r}_{\sigma(i)}$. Due to periodicity of $\omega_{i}$, the images of these eigenbranes are located at:

$$
\mathbf{r}_{i}\left(n_{w}\right)=\left(L^{-1}\left(\omega_{i}+2 \pi n_{w}\right), v_{i}\right), \quad n_{w} \in \mathbb{Z} .
$$


The utility of this description is that it geometrizes various aspects of monopole-instantons at small $\mathbb{S}^{1}$. The action of the monopole-instantons associated with magnetic charge $\alpha_{i}$ embedded into $\mathbb{R}^{3} \times \mathbb{S}^{1}$ is:

$$
S_{i}=\frac{4 \pi L}{g_{4}^{2}}\left|\mathbf{r}_{i}(0)-\mathbf{r}_{i+1}(0)\right|=\frac{4 \pi L}{g_{4}^{2}} \sqrt{\left(v_{i}-v_{i+1}\right)^{2}+L^{-2}\left(\omega_{i}-\omega_{i+1}\right)^{2}}
$$

and the action of the winding 3d instantons (the Kaluza-Klein tower of (B.6)) is:

$$
\begin{aligned}
S_{i, n_{w}} & =\frac{L}{g_{4}^{2}}\left|\mathbf{r}_{i}(0)-\mathbf{r}_{i+1}\left(n_{w}\right)\right| \\
& =\frac{4 \pi L}{g_{4}^{2}} \sqrt{\left(v_{i}-v_{i+1}\right)^{2}+L^{-2}\left(\omega_{i}-\omega_{i+1}+2 \pi n_{w}\right)^{2}}, \quad n_{w} \in \mathbb{Z} .
\end{aligned}
$$

This formula is noting but the distance between $\mathbf{r}_{i}(0)$ and the image of $\mathbf{r}_{i+1}(0)$ labeled as $\mathbf{r}_{i+1}\left(n_{w}\right)$.

The fugacity function associated with monopole-instantons of charge $\alpha_{i}$ is therefore, similar to eq. (3.24):

$$
F_{i}\left(\omega_{i}-\omega_{i+1}\right)=\sum_{n_{w} \in \mathbb{Z}} e^{-S_{i, n_{w}}}=\sum_{n_{w} \in \mathbb{Z}} e^{-\frac{4 \pi L}{g_{4}^{2}} \sqrt{\left(v_{i}-v_{i+1}\right)^{2}+L^{-2}\left(\omega_{i}-\omega_{i+1}+2 \pi n_{w}\right)^{2}}} .
$$

We should note that all the instantons in the tower have the same magnetic charge, but their topological charges differ by one unit. The part of the sum contributing to $n_{w} \geq 0$ can smoothly be deformed to the self-dual monopole-instantons by taking $\left(v_{i}-v_{i+1}\right)=0$, whereas under the same smooth deformation, the $n_{w} \leq-1$ terms arise from non-selfdual monopole-instantons. As it was the case in $\mathrm{SU}(2)$, the sum over the winding number combines self-dual and anti-selfdual monopole-instantons into a single tower. In the sense of magnetic charge, this is possible because the simple roots $\left\{\alpha_{1}, \alpha_{2}, \ldots, \alpha_{N-1}\right\}$ and affine $\operatorname{root}\left\{\alpha_{N}\right\}$ of $\mathrm{SU}(N)$ satisfy $\alpha_{i}=\alpha_{i}+n_{w} \sum_{j=1}^{N} \alpha_{j}$.

Since the function $F_{i}\left(\omega_{i}-\omega_{i+1}\right)$ from (B.8) is periodic in $\omega_{i}-\omega_{i+1}$, we can Fourier expand it and follow the steps given in section 3.5. The result is

$$
F_{i}\left(\omega_{i}-\omega_{i+1}\right)=\sum_{n_{e} \in \mathbb{Z}} \frac{v L}{\pi} \cos \delta_{n_{e}} K_{1}\left(L M\left(1, n_{e}\right)\right) e^{i n_{e}\left(\omega_{i}-\omega_{i+1}\right)},
$$

where $M\left(1, n_{e}\right)$ is the mass formula for dyon particles given in (B.1). This is the generalization of Poisson duality between the tower of $3 \mathrm{~d}$ monopole-instantons and the $4 \mathrm{~d}$ tower of dyonic excitations of a monopole.

Open Access. This article is distributed under the terms of the Creative Commons Attribution Noncommercial License which permits any noncommercial use, distribution, and reproduction in any medium, provided the original author(s) and source are credited.

\section{References}

[1] N. Seiberg and E. Witten, Monopole condensation, and confinement in $N=2$ supersymmetric Yang-Mills theory, Nucl. Phys. B 426 (1994) 19 [Erratum ibid. B 430 (1994) 485] [hep-th/9407087] [SPIRES]. 
[2] N. Seiberg and E. Witten, Gauge dynamics and compactification to three dimensions, hep-th/9607163 [SPIRES].

[3] M. Ünsal, Abelian duality, confinement, and chiral-symmetry breaking in a SU(2) QCD-like theory, Phys. Rev. Lett. 100 (2008) 032005 [arXiv:0708.1772] [SPIRES].

[4] M. Ünsal, Magnetic bion condensation: a new mechanism of confinement and mass gap in four dimensions, Phys. Rev. D 80 (2009) 065001 [arXiv:0709.3269] [SPIRES].

[5] A.M. Polyakov, Quark confinement and topology of gauge groups, Nucl. Phys. B 120 (1977) 429 [SPIRES].

[6] T.M.W. Nye and M.A. Singer, An $L^{2}$-index theorem for Dirac operators on $\mathbb{S}^{1} \times \mathbb{R}^{3}, J$. Funct. Anal. 177 (2000) 203 [math/0009144] [SPIRES].

[7] E. Poppitz and M. Ünsal, Index theorem for topological excitations on $\mathbb{R}^{3} \times \mathbb{S}^{1}$ and Chern-Simons theory, JHEP 03 (2009) 027 [arXiv:0812.2085] [SPIRES].

[8] E.J. Weinberg and P. Yi, Magnetic monopole dynamics, supersymmetry and duality, Phys. Rept. 438 (2007) 65 [hep-th/0609055] [SPIRES].

[9] A.S. Kronfeld, G. Schierholz and U.J. Wiese, Topology and dynamics of the confinement mechanism, Nucl. Phys. B 293 (1987) 461 [SPIRES].

[10] K.-M. Lee and P. Yi, Monopoles and instantons on partially compactified D-branes, Phys. Rev. D 56 (1997) 3711 [hep-th/9702107] [SPIRES].

[11] T.C. Kraan and P. van Baal, Periodic instantons with non-trivial holonomy, Nucl. Phys. B 533 (1998) 627 [hep-th/9805168] [SPIRES].

[12] H. Nishimura and M.C. Ogilvie, Phase structure of confining theories on $\mathbb{R}^{3} \times \mathbb{S}^{1}$, AIP Conf. Proc. 1343 (2011) 167 [arXiv:1012.0333] [SPIRES].

[13] N. Dorey, Instantons, compactification and S-duality in $N=4$ SUSY Yang-Mills theory. I, JHEP 04 (2001) 008 [hep-th/0010115] [SPIRES].

[14] N. Dorey and A. Parnachev, Instantons, compactification and S-duality in N $=4$ SUSY Yang-Mills theory. II, JHEP 08 (2001) 059 [hep-th/0011202] [SPIRES].

[15] H.-Y. Chen, N. Dorey and K. Petunin, Wall crossing and instantons in compactified gauge theory, JHEP 06 (2010) 024 [arXiv: 1004.0703] [SPIRES].

[16] J. Zinn-Justin, Quantum field theory and critical phenomena, Int. Ser. Monogr. Phys. 113 (2002) 1 [SPIRES].

[17] P. Cvitanović, R. Artuso, R. Mainieri, G. Tanner and G. Vattay, Chaos: classical and quantum, Niels Bohr Institute, Copenhagen (2009) http://www.chaosbook.org/.

[18] D. Gaiotto, G.W. Moore and A. Neitzke, Four-dimensional wall-crossing via three-dimensional field theory, Commun. Math. Phys. 299 (2010) 163 [arXiv:0807.4723] [SPIRES].

[19] M.R. Douglas and S.H. Shenker, Dynamics of $\mathrm{SU}(N)$ supersymmetric gauge theory, Nucl. Phys. B 447 (1995) 271 [hep-th/9503163] [SPIRES].

[20] M.J. Strassler, An unorthodox introduction to supersymmetric gauge theory, hep-th/0309149 [SPIRES].

[21] O. Aharony, A. Hanany, K.A. Intriligator, N. Seiberg and M.J. Strassler, Aspects of $N=2$ supersymmetric gauge theories in three dimensions, Nucl. Phys. B 499 (1997) 67 [hep-th/9703110] [SPIRES]. 
[22] I. Affleck, J.A. Harvey and E. Witten, Instantons and (super)symmetry breaking in (2+1)-dimensions, Nucl. Phys. B 206 (1982) 413 [SPIRES].

[23] N. Dorey, V.V. Khoze, M.P. Mattis, D. Tong and S. Vandoren, Instantons, three-dimensional gauge theory and the Atiyah-Hitchin manifold, Nucl. Phys. B 502 (1997) 59 [hep-th/9703228] [SPIRES].

[24] N.M. Davies, T.J. Hollowood, V.V. Khoze and M.P. Mattis, Gluino condensate and magnetic monopoles in supersymmetric gluodynamics, Nucl. Phys. B 559 (1999) 123 [hep-th/9905015] [SPIRES].

[25] N.M. Davies, T.J. Hollowood and V.V. Khoze, Monopoles, affine algebras and the gluino condensate, J. Math. Phys. 44 (2003) 3640 [hep-th/0006011] [SPIRES].

[26] M.M. Anber and E. Poppitz, Microscopic structure of magnetic bions, JHEP 06 (2011) 136 [arXiv: 1105.0940] [SPIRES].

[27] E. Poppitz and M. Ünsal, Conformality or confinement: (IR)relevance of topological excitations, JHEP 09 (2009) 050 [arXiv:0906.5156] [SPIRES].

[28] E. Poppitz and M. Ünsal, Conformality or confinement (II): one-flavor CFTs and mixed-representation QCD, JHEP 12 (2009) 011 [arXiv:0910.1245] [SPIRES].

[29] P. Argyres and M. Ünsal, Confinement on $\mathbb{R}^{3} \times \mathbb{S}^{1}$ for arbitrary gauge groups with adjoint fermions, work in preparation.

[30] E. Poppitz and M. Ünsal, Thermal bions, Possion resummation, and deconfinement, work in preparation.

[31] J.A. Harvey, Magnetic monopoles, duality and supersymmetry, hep-th/9603086 [SPIRES].

[32] D. Diakonov, Topology and confinement, Nucl. Phys. Proc. Suppl. 195 (2009) 5 [arXiv:0906.2456] [SPIRES].

[33] E.B. Bogomolny, Calculation of instanton-anti-instanton contributions in quantum mechanics, Phys. Lett. B 91 (1980) 431 [SPIRES].

[34] J. Zinn-Justin, Multi-instanton contributions in quantum mechanics, Nucl. Phys. B 192 (1981) 125 [SPIRES].

[35] I.I. Balitsky and A.V. Yung, Instanton molecular vacuum in $N=1$ supersymmetric quantum mechanics, Nucl. Phys. B 274 (1986) 475 [SPIRES].

[36] A.V. Yung, Large distance behavior of supersymmetric QCD and instanton vacuum, Nucl. Phys. B 344 (1990) 73 [SPIRES].

[37] M. Ünsal and L.G. Yaffe, Center-stabilized Yang-Mills theory: confinement and large- $N$ volume independence, Phys. Rev. D 78 (2008) 065035 [arXiv:0803.0344] [SPIRES].

[38] J. Greensite, An introduction to the confinement problem, Lect. Notes Phys. 821 (2011) 1 [SPIRES]. 